\title{
Axi-symmetric models of ultraviolet radiative transfer with applications to circumstellar disk chemistry
}

\author{
G.-J. van Zadelhoff ${ }^{1}$, Y. Aikawa ${ }^{2}$, M. R. Hogerheijde ${ }^{3}$, and E. F. van Dishoeck ${ }^{1}$ \\ ${ }^{1}$ Leiden Observatory, PO Box 9513, 2300 RA Leiden, The Netherlands \\ 2 Dept. of Earth and Planetary Sciences, Kobe University, 1-1 Rokkoudai, Nada-ku, Kobe, 657-8501, Japan \\ 3 Steward Observatory, The University of Arizona, 933 N. Cherry Ave. Tucson AZ, USA
}

Received 8 April 2002 / Accepted 29 October 2002

\begin{abstract}
A new two-dimensional axi-symmetric ultraviolet radiative transfer code is presented, which is used to calculate photodissociation and ionization rates for use in chemistry models of flaring circumstellar disks. Scattering and absorption of photons from the central star and from the interstellar radiation field are taken into account. The molecules are effectively photodissociated in the surface layer of the disk, but can exist in the intermediate, moderately warm layers. A comparison has been made with an approximate $2 \mathrm{D}$ ray-tracing method and it was found that the latter underestimates the ultraviolet field and thus the molecular photodissociation rates below the disk surface. The full 2D results show significantly higher abundances of radicals such as $\mathrm{CN}$ and $\mathrm{C}_{2} \mathrm{H}$ than previous work, partly due to the fact that $\mathrm{CO}$ is dissociated to greater depths. Results for different stellar radiation fields are also presented. The $\mathrm{CN} / \mathrm{HCN}$ ratio shows a strong dependence on the stellar spectrum, whereas other ratios such as $\mathrm{HCO}^{+} / \mathrm{CO}$ show only little variation.
\end{abstract}

Key words. ISM: molecules - stars: pre-main sequence - stars: circumstellar matter - stars: planetary systems: protoplanetary disks - radiative transfer - line: profiles

\section{Introduction}

Numerous recent observations of dust and molecular line emission have increased the understanding of disks around isolated T Tauri stars in both their appearances and internal structure (Beckwith \& Sargent 1996; Dutrey et al. 2000; Qi 2001). The modeling to date can be divided in two categories. A first category deals with the physical structure of disks. It was proposed by Kenyon \& Hartmann (1987) that disks have a flared geometry, where the stellar light puffs up the outer regions of the disk. Recent papers by Chiang \& Goldreich (1997, 1999), Men'shchikov \& Henning (1997), D'Alessio et al. (1998, 1999), Dullemond et al. (2002) and Nomura (2002) show similar results by calculating explicitly the density and temperature distributions in disks in hydrostatic equilibrium. Chiang \& Goldreich (1997) fit spectral energy distributions by considering disks with a bi-layered temperature structure. This structure is calculated assuming that the ultraviolet and visible light from the star heats up the outer layer of the disk, and does not effect the shielded region directly. D'Alessio et al. (1998) calculate the full temperature structure in the vertical direction and include the effects of accretion and viscous heating.

The second category takes a physical disk model and uses a time-dependent chemistry code to calculate the molecular

Send offprint requests to: G. J. van Zadelhoff, e-mail: zadelhof@knmi.nl abundances in the gas (Aikawa et al. 1997; Aikawa \& Herbst 1999; Willacy et al. 1998; Willacy \& Langer 2000). Aikawa \& Herbst (1999) use the minimum-mass solar nebula model of Hayashi (1981), whereas Willacy et al. (2000) use the Chiang \& Goldreich model. In the first model, the temperatures are very low throughout the disk, thereby freezing out the molecules onto grains, while in the latter model nearly all molecules are dissociated due to the ultraviolet light in the upper layer and are depleted in the cold lower layer. Both models have to assume either an artificially low sticking probability or a very high photo-desorption rate to keep molecules in the gas.

In the latest paper by Aikawa et al. (2002, hereafter Paper I), the D'Alessio et al. model was used to calculate molecular abundances. The stellar and interstellar ultraviolet (UV) radiation heats the dust and gas, and dissociates molecules in the surface region of $A_{v} \lesssim 1$ mag. In the deeper layers, visible photons with lower energies are available to heat the grains and gas, but there are much fewer ultraviolet photons for dissociation than in the surface layer. The dust in the surface layer, heated by the ultraviolet and visible photons, in turn emits infrared photons, which can travel even further toward the mid-plane until they are eventually absorbed. Viscous heating is only important in the inner few AU of the disk, which are not considered in this study. As a result, the disk has three distinctive chemical layers. In the surface layer with temperatures $T>40 \mathrm{~K}$ and density $n_{\mathrm{H}} \sim 10^{4}-10^{5} \mathrm{~cm}^{-3}$, molecules 
are mostly dissociated. In the intermediate layer, conditions are comparable to those in dense interstellar clouds, i.e., the temperature is $20-40 \mathrm{~K}$ and density is $\sim 10^{6}-10^{7} \mathrm{~cm}^{-3}$. This layer contains sufficient amounts of gaseous molecules to reproduce the observations of some disks. In the innermost layer including the midplane, the density is high $\left(n_{\mathrm{H}}>10^{7} \mathrm{~cm}^{-3}\right)$ and temperature is low $(T \lesssim 20 \mathrm{~K}$ ). Nearly all the gaseous species, except for $\mathrm{H}_{2}$ and $\mathrm{He}$, freeze out onto the grains and stay there until the grains are again heated, e.g. when most of the disk has dissipated and becomes optically thin to ultraviolet radiation or when the grains are dynamically transported to a warmer part of the disk.

In this work, we again adopt the density and temperature distribution of the D'Alessio et al. model, and use a new twodimensional (2D) ultraviolet radiative transfer code to describe the radiative effects on the chemistry. Previous works, including our Paper I, treated the UV and optical radiative transfer in disks in a one dimensional (1D) or approximate 2D method. In the former case it is assumed that the disks are geometrically thin, i.e. the scale-height of the disk is small compared to the distance to the star, and therefore radiative transfer in the radial direction is negligible compared to that in the vertical direction (Chiang \& Goldreich 1997; D'Alessio et al. 1998). In the latter case the attenuation both along the line of sight from the star and in the vertical direction is calculated (Paper I). This method cannot treat scattering effects in principle and therefore only gives an approximate depth-dependent intensity; forward scattering will help stellar photons at UV and optical wavelengths to penetrate deeper into the disk, which will affect the chemistry through photodissociation. One important aim of this paper is a direct assessment of the effects of the correct 2D treatment compared with the earlier more approximate methods. The $2 \mathrm{D}$ radiative transfer code also enables us to consider dissociation of $\mathrm{H}_{2}$ and $\mathrm{CO}$ via the stellar $\mathrm{UV}$ radiation, which was neglected in Paper I. In addition the dependence of molecular abundances and line intensities on the stellar spectrum can be studied.

Paper I and this paper are complementary to the recent work of Markwick et al. (2002). Whereas our studies focus on the outer regions of the disk ( $>50 \mathrm{AU}$ ), Markwick et al. (2002) consider the inner $10 \mathrm{AU}$ in a quasi-static treatment; the chemistry is calculated locally, with the starting conditions defined by the chemical abundances calculated at more distant radial points. Their calculations confirm the results of earlier 1D studies that the chemistry in the inner region is driven by thermal desorption. The inclusion of $\mathrm{X}$-rays in their model shows that radicals such as $\mathrm{CN}$ and $\mathrm{C}_{2} \mathrm{H}$ are important tracers of the ionization in disks, a conclusion also reached for the outer disks in our papers.

The outline of this paper is as follows. In Sect. 2, the coupling between the radiation and chemistry is discussed. In Sect. 3 the results are presented of the inclusion of continuum radiative transfer on the chemistry and compared with the approximate 2D method. The differences in line strength of the various molecules are calculated in Sect. 4. Discussion is in Sect. 5 , followed by a conclusion in Sect. 6 . Finally the adopted 2D Monte Carlo UV radiative transfer code is explained in detail in Appendix A.

\section{Chemistry and ultraviolet radiative transfer}

\subsection{General considerations}

The gas-phase molecular abundances are governed by two different effects of the radiation. On the one hand, the stellar radiation heats up the disk, assuring that freeze-out onto grains does not occur. This heating by direct radiation is dominated by the lower energy photons emitted by the cool central star $\left(T_{*}=4000 \mathrm{~K}\right)$. In this paper, we assume that the gas and dust temperatures are coupled and adopt the calculated dust temperatures from D'Alessio et al. (1999). On the other hand, the higher-energy UV radiation dissociates and/or ionizes molecular and atomic species and most $\mathrm{T}$ Tauri stars have a relatively large UV flux compared to main sequence stars of the same temperature (e.g. Costa et al. 2000). The mean intensity of the visible and ultraviolet light at a position in the disk is controlled by absorption and scattering, making the solution non-trivial. In this section, we briefly discuss the UV radiative transfer in the disk. The numerical details of the Monte Carlo code are explained in more detail in Appendix A.

Before combining the radiative transfer with the chemistry, the different time scales in the problem are checked. The three time scales of interest are: the radiative time scale, the chemical time scale, and the dynamical time scale. Since the current line observations of disks have poor spatial resolution, even for sources mapped with millimeter interferometers (Dutrey et al. 1996; Qi 2001), they are mostly sensitive to the outer regions of the disk. A comparison of the time scales is therefore most appropriate at distances $\geq 100 \mathrm{AU}$ from the star.

The first time scale represents the time needed for the radiation field to adapt to fluctuations in physical and/or chemical processes. The disk itself is too cold to emit ultraviolet or visible photons, therefore the mean intensity at each point in the disk depends only on changes in the source, both stellar or interstellar, and the density distribution in between the source and that point. The radiation field reaches its mean value on the same time scale as it takes the light to travel from the source to the point of interest; for 100 AU this is $\sim 14$ hours from the star. So the radiation field is always in equilibrium in the time scales we are concerned with in this paper.

The chemical time scale, i.e., the time in which the number density of a species reaches its steady-state value, varies with molecular species and position in the disk. In the intermediate molecular layer we are concerned with, it is of the order of $10^{4}$ $10^{5}$ years for many organic molecules (Paper I). On the other hand, in the midplane layer the time scale of adsorption, which is the dominant process, is very short: $\sim 10^{2}\left(10^{8} \mathrm{~cm}^{-3} / n_{\mathrm{H}}\right) \mathrm{yr}$.

There are several dynamical time scales in the disk. The typical accretion time scale of the disk as a whole is estimated to be $10^{6} \mathrm{yr}$, derived from dividing the disk mass $\left(10^{-2} M_{\odot}\right)$ by the mass accretion rate $\left(10^{-8} M_{\odot} \mathrm{yr}^{-1}\right)$. The local accretion timescale at $R=100 \mathrm{AU}$, i.e. the time it takes for a parcel of gas to move $5 \mathrm{AU}$ (less than the radial gridsize in our model) radially inwards, is estimated to be shorter, $7 \times 10^{4} \mathrm{yr}$ from the local radial drift velocity $v_{\mathrm{R}}=\dot{M} /[2 \pi R \Sigma(R)]$. Since accretion is caused by the transport of angular momentum due to turbulence, the timescale for turbulent mixing is also relevant. 
This timescale for radial mixing over $\Delta R$ is $\sim(\Delta R)^{2} /\left(l_{\text {turb }} b_{\text {turb }}\right)$, for $\Delta R$ larger than the size of turbulent eddy $l_{\text {turb }}$ (e.g. Aikawa et al. 1996). The actual Eddy size $\left(l_{\text {turb }}\right)$ and turbulent velocity $\left(v_{\text {turb }}\right)$ are highly uncertain, but if they are assumed to be $10 \%$ of the vertical scale height and sound velocity, the timescale for global mixing (i.e. $\Delta R \sim R \sim 100 \mathrm{AU}$ ) is of the order of $10^{6} \mathrm{yr}$. The local mixing timescale with smaller $\Delta R$ (or $\Delta Z$ ) is accordingly shorter.

In this paper we assume that the disk is static; the molecular abundances are calculated at each point $(R, Z)$ in the disk, without considering any accretion flow or mixing for simplicity. Although this static model is invalid from a physical point of view, as the comparison of the different local time-scales show above, this simplification is adopted for the following two reasons. First, disk chemistry is a complicated mixture of various chemical processes and physical processes (e.g., density and temperature distributions, radiative transfer and hydrodynamics). In this series of papers, the importance of each of the processes is investigated step by step; in Paper I the chemical processes which are important in the different regions of the disk were analyzed, and in the current paper the effect of 2dimensional UV radiative transfer is investigated. Mixing and accretion smooth out the molecular stratification over some length, determined by the balance between the chemical and dynamical time scales. At the same time radical species are transfered in the intermediate layer, activating chemical reactions in neighbouring regions. These effects are expected to be larger in the vertical direction than in the radial direction, since the radial distributions do not vary much in the outer disk between 50 and $400 \mathrm{AU}$ (Paper I). They should be investigated in detail in independent future work. Second, full coupling of these effects in 2- or 3-dimensional calculations would be very complicated and too time-consuming with current computational facilities. In order to accomplish the full coupling of chemistry, radiative transfer and (magneto-) hydrodynamics, the chemical reaction network needs to be significantly reduced by carefully choosing the dominant chemical reactions in each region of the disk. The results presented in our papers using a large chemical network will assist the construction and testing of future coupled chemical-hydrodynamical models.

Another relevant physical process is the settling of dust and grain-growth. In this paper we assume for simplicity that the optical properties of dust grains are the same as those of interstellar clouds, and that the gas and dust are well mixed. Settling of small (sub-micron sized) grains, which are the main source of the UV opacity, takes place over a period of at least $10^{6}$ years (e.g. Nakagawa et al. 1981; Weidenschilling 1997). The coagulation timescale, however, is much shorter. In fact, the spectral energy distributions (SEDs) of T Tauri stars indicate significant increase to $\mathrm{mm}$-sized grains compared with interstellar dust (Chiang et al. 2001; D'Alessio et al. 2001). Inclusion of dust coagulation in our radiation-chemistry disk model is however more complicated, since variations in dustproperties, scattering and possible decoupling of the gas and dust temperatures could be of essential importance. In previous work on the vertical disk structure, the temperature distribution is determined by radiative transfer, with dust as the main source of opacity, whereas the density distribution is determined by the hydrostatic equilibrium, assuming the same gas and dust temperatures. If the number of small grains decreases due to coagulation, the two temperatures may decouple if not enough collisions between gas and dust particles take place. Calculations of the decoupled gas and dust temperature have only been performed for tenuous disks which have dust masses that are at least two orders of magnitude less than the disks studied in this work and are optically thin to UV and optical radiation, simplifying the radiative transfer (Kamp \& van Zadelhoff 2001). This problem of dust coagulation is left for future work, and a basic $2 \mathrm{D}$ radiation-chemistry model with interstellar-sized grains is established in this paper.

The chemical model adopted in this paper is the "new standard model" (NSM) for the gas-phase chemistry (Tervieza \& Herbst 1998; Osamura et al. 1999), extended to include deuterium chemistry, adsorption of molecules onto grains, and thermal desorption (Aikawa \& Herbst 1999; and references therein). Chemical processes by X-rays are not included in this paper.

\subsection{Impact of ultraviolet radiation}

The chemical abundances are related through a reaction network. In this network the dissociation- and ionization-rates $k_{\mathrm{i}}$ of the different species are calculated using

$k_{\mathrm{i}}=\int_{0}^{\infty} 4 \pi \frac{\lambda}{h c} \sigma_{\mathrm{i}}(\lambda) J_{\lambda} \mathrm{d} \lambda$

where $h$ is the Planck constant, $c$ the velocity of light and $J_{\lambda}$ the mean intensity at wavelength $\lambda$, defined as

$J_{\lambda}=\frac{1}{4 \pi} \int_{\Omega} I_{\lambda} \mathrm{d} \Omega$.

Some molecules can be dissociated by absorption of photons over a continuous part of the spectrum, while others need discrete line transitions or a combination of the two (van Dishoeck 1988). The cross sections $\sigma_{\mathrm{i}}(\lambda)$ or oscillator strengths $f_{\mathrm{i}}(\lambda)$ need to be specified for each transition for each molecule. We adopt here the values given by van Dishoeck (1988), updated by Jansen et al. (1995a, 1995b). For species for which no data on the cross sections are available, a rate given by a similar type of molecule was adopted.

The photodissociation rate of a species depends on the mean intensity of the radiation in the spatial grid, which is obtained via the 2D Monte Carlo code. This code is based on the same principles as those developed by Boissé (1990), Spaans (1996) and Gordon et al. (2001) for interstellar applications and e.g., Whitney \& Hartmann (1992) for circumstellar disks. In general, one needs to define a source with a surface area $S$, where the total energy is the flux from the source times the surface area. The total energy is divided into a number of photonpackages $\left(N_{\lambda}\right)$, which then travel through the grid. The energy density at each point is calculated using both the number of photons passing through a grid cell and the mean energy transported by each photon during its transfer. Photon packages are either absorbed or scattered by dust grains and are followed until they leave the system or have reached a minimum intensity. Optical properties of dust grains are assumed to be the 
same as those of interstellar dust; the scattering is described by the Henyey-Greenstein probability function (Eq. (A.8)), and albedo and opacities are taken from Roberge et al. (1991).

In the Monte Carlo method, the radiation field within the disk is readily obtained for irradiation by both stellar and interstellar radiation: since each source of radiation gives its own energy density, calculations for different sources can be added to give a total energy density. In addition, radiation fields for different stellar spectra are easily calculated (Sect. 3.4). The energy densities in the disk are directly proportional to the emitted stellar flux, and are thus scalable. Since the disk structure is independent on the precise UV spectrum of the central star, a single calculation is sufficient for obtaining the full UV radiation distribution for each assumed spectrum.

In the 912-1110 ̊ region, additional attenuation due to $\mathrm{H}_{2}$ and $\mathrm{CO}$ self-shielding was included. The shielding of other species by $\mathrm{H}_{2}$ was ignored in these calculations. This could lead to an overestimate of dissociation rates for species which dissociate only in the 912-1110 A region by at least $20 \%$ (Draine \& Bertoldi 1996). Self-shielding of $\mathrm{H}_{2}$ and $\mathrm{CO}$ depends on the column densities of the molecules themselves along the photon trajectories as well as the dust attenuation $\left(A_{\mathrm{v}}\right)$, which means that the chemical abundances and the UV radiative transfer need to be solved simultaneously. For the interstellar UV field this is easily done via a 1D slab model (van Dishoeck \& Black 1988; Lee et al. 1996), which is adopted in this paper and previous works (e.g. Paper I). Shielding of the stellar UV radiation is more difficult to calculate, since iteration of the chemistry and $2 \mathrm{D}$ radiative transfer is very time-consuming. Since we are not interested in the precise position of the $\mathrm{H}-\mathrm{H}_{2}$ transition, this iteration is performed only once: we first calculate the mean intensity assuming that all hydrogen is in molecular form and adopt the shielding factor given by Draine \& Bertoldi (1996), their formula 37 (A.13, see Sect. A.3 for details), and then obtain the $\mathrm{H}_{2}$ abundance from chemical network. The selfshielding of $\mathrm{CO}$ has been calculated in a similar way using the shielding functions from Lee et al. (1996).

\section{Results}

\subsection{UV radiation and photodissociation rates}

The temperature and density structure of the disk model by D'Alessio et al. (1999) is adopted as our fiducial model, which was calculated for an accretion rate $\dot{M}=10^{-8} M_{\odot} \mathrm{yr}^{-1}$ and viscosity parameter $\alpha=0.01$. The disk has a radius of $400 \mathrm{AU}$ and a mass of $0.05 M_{\odot}$, representative of the disk around the T Tauri star LkCa15 for which molecular data exist (Qi 2001; van Zadelhoff et al. 2001; Thi et al. 2002). Similar sizes or even larger have been observed for a number of gas disks, see, e.g., Simon et al. (2001): LkCa 15 (650 AU; 435 AU by Qi 2001), DM Tau (800 AU), DL Tau (520 AU) and GM Aur (525 AU). These are the same disks for which observations of molecules other than $\mathrm{CO}$ are available and for which chemical models have been presented in Paper I. The adopted disk model is gravitationally stable according to the Toomre criterion up to at least $340 \mathrm{AU}$ (D'Alessio et al. 1999, see also Bell et al. 1997 for a discussion on this point). We therefore truncated our disk at the radial grid cell in which this radius is reached, $R_{\text {out }}=400 \mathrm{AU}$.

The disk structure is calculated self-consistently for a stellar temperature of $4000 \mathrm{~K}$ and $R_{*}=2 R_{\odot}$ (D'Alessio et al. 1999). The disk is assumed to be subjected to UV radiation from the interstellar radiation field and from the star. The Draine (1978) field is used to represent the interstellar field between 912 and $2000 \AA$, extended to longer wavelengths as specified by van Dishoeck \& Black (1982). For the star, initially the same spectral shape is taken, but scaled by a factor such that the intensity at $R=100 \mathrm{AU}$ incident on the surface of the disk is a factor of $10^{4}$ higher than the interstellar field. This choice was motivated by Herbig \& Goodrich (1986) and was also adopted in Paper I. In Sect. 3.4, different assumptions for the stellar radiation field are explored. It is assumed that the additional UV radiation does not change the disk structure. The integrated flux in the range $912-4000 \AA$, - the range important for the chemistry -, can contribute to a maximum of $10 \%$ of the total energy for the stars modeled in this paper. The UV radiative transfer was calculated using the grain properties and extinction curve by Weingartner \& Draine (2001).

Figure 1 shows the resulting distribution of the UV intensity in the disk for two different wavelengths, with overplotted the density and temperature structure. It is seen that for longer wavelengths, the radiation penetrates deeper into the disk because of the reduced absorption. Figure 2 shows the UV spectrum at various heights at $R=105 \mathrm{AU}$. Even at short wavelengths (912-2000 $\mathrm{A}$ ), the intensity is still comparable to the unattenuated interstellar radiation field down to $35 \mathrm{AU}$ at $R \approx 100 \mathrm{AU}$. The longer wavelengths visible photons start to dominate the radiation field below $50 \mathrm{AU}$. The dip at $\lambda \sim 2200 \AA$ is caused by the bump in the extinction curve.

The effect of radiation on the molecular abundances is reflected in the different dissociation or ionization rates. For example, Fig. 3 shows the dissociation rates of $\mathrm{C}_{2} \mathrm{H}\left(\mathrm{C}_{2} \mathrm{H} \rightarrow\right.$ $\left.\mathrm{C}_{2}+\mathrm{H}\right)$ and $\mathrm{H}_{2} \mathrm{CO}\left(\mathrm{H}_{2} \mathrm{CO} \rightarrow \mathrm{CO}+\mathrm{H}+\mathrm{H}\right) \cdot \mathrm{C}_{2} \mathrm{H}$ is dissociated by lines in between $1080-1530 \AA$ and $\mathrm{H}_{2} \mathrm{CO}$ is dissociated due to both line (1100-1749 $\AA$ ) and continuum radiation (912-1505 $\AA$ ). The solid lines show the rates calculated from the stellar and interstellar radiation field, obtained via the full $2 \mathrm{D}$ radiative transfer. The dotted and dashed lines show the rates calculated using the depth-dependent dissociation rates of van Dishoeck (1988) and NSM, respectively. Van Dishoeck (1988) calculated a plane-parallel PDR model, and obtained the dissociation rates $k$ as functions of depth from the cloud surface. These were fitted by the single exponential decay function:

$k=k_{0} \mathrm{e}^{-\beta A_{\mathrm{v}}}$.

The NSM assumes the same function, but with slightly different coefficients. In Aikawa \& Herbst (1999) and Paper I, the visual extinction $A_{\mathrm{v}}$ from the central star is obtained at each position in the disk by integrating the density distribution along the line of sight to the central star, and the dissociation rate is obtained with the NSM depth-dependent rates. The dissociation rate via the interstellar UV field is obtained in a similar way using the depth-dependent NSM rates and calculating $A_{\mathrm{v}}$ from the disk surface. Figure 3 shows the total dissociation 

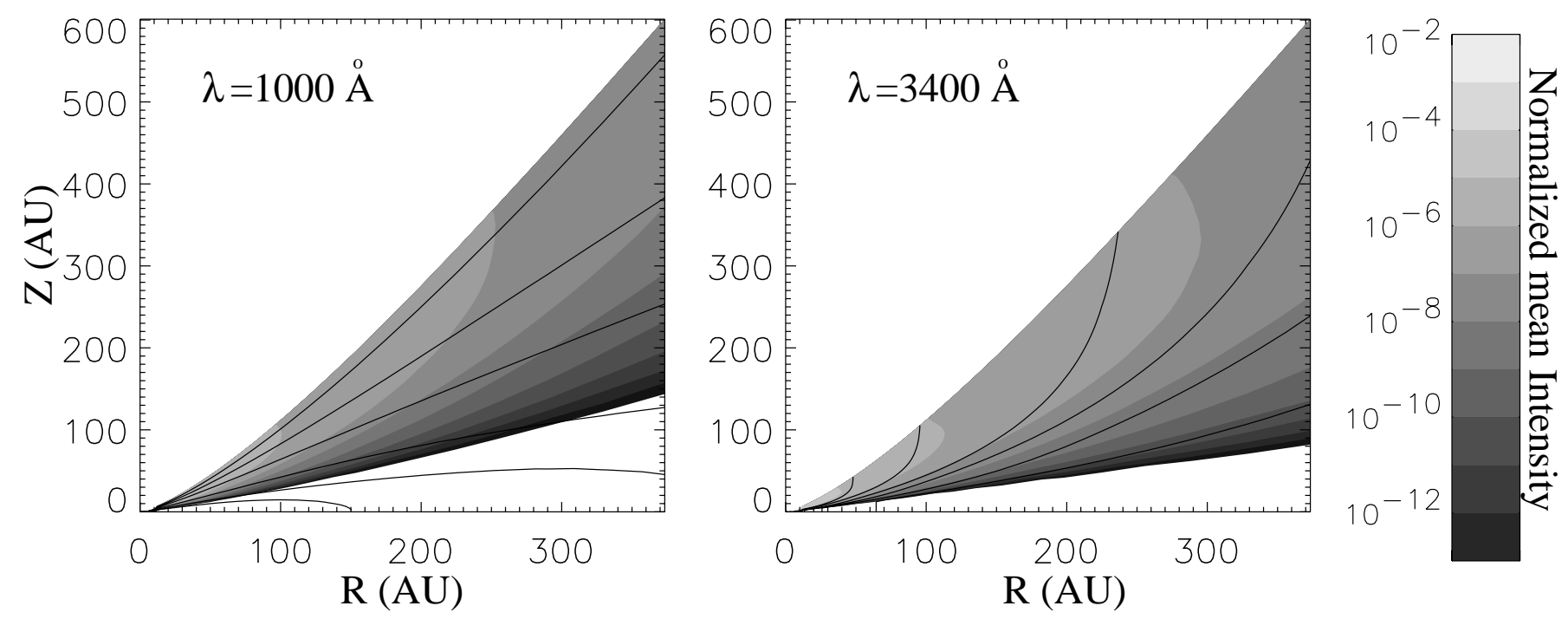

Fig. 1. Normalized mean intensities at 1000 and $3400 \AA$ for the adopted model. The mean intensities have been normalized to the value in the innermost cell to better visualize the effects of grain scattering and absorption. Overplotted are the $\mathrm{H}_{2}$ number density contours (from surface to midplane: $5 \times 10^{4}, 2 \times 10^{5}, 10^{6}, 10^{7}, 10^{8}, 10^{9}$ ) $\left[\mathrm{cm}^{-3}\right]$ on the left and the dust temperature contours (upper left to lower right: $90,70,50,40$, $30,20)[\mathrm{K}]$ on the right. Only one quadrant of the $2 \mathrm{D}$ flaring disk is shown.

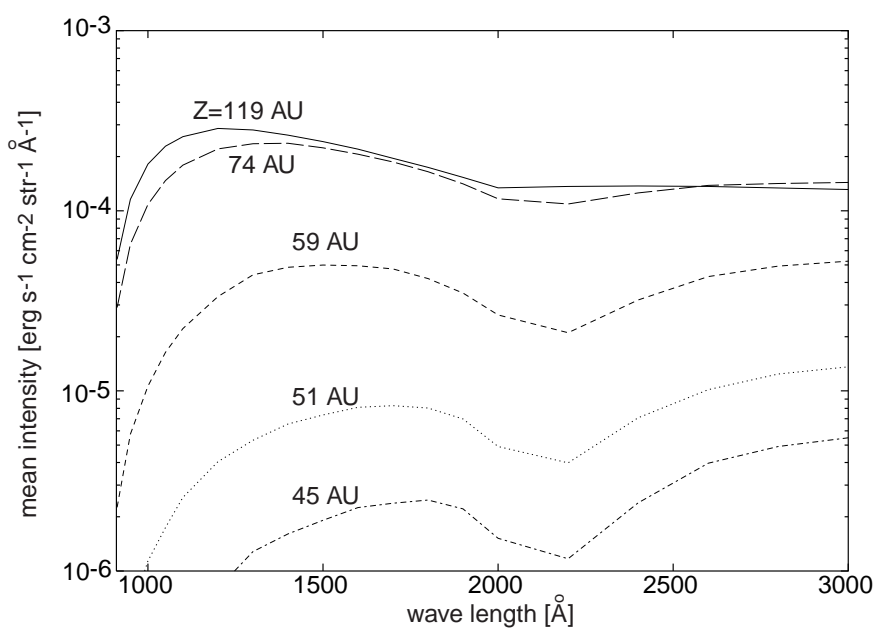

Fig. 2. Spectrum of the mean intensity $J_{\lambda}$ at different heights in the disk for a radius of $R=105 \mathrm{AU}$. Numerals in the figure indicate the height from the midplane. The stellar UV is assumed to be $10^{4}$ times higher than interstellar field at $R=100 \mathrm{AU}$ and have the same shape.

rate via UV radiation from the interstellar field and the central star. In the dotted and dashed lines, the two components can be easily distinguished: radiation from the central star dominates at $Z \gtrsim 50 \mathrm{AU}$, while interstellar radiation dominates at $Z \lesssim 50 \mathrm{AU}$.

In Fig. 3, it is seen that the dissociation rates are significantly underestimated at $Z \sim 50 \mathrm{AU}$, if the approximate depthdependent rates described above are used. This is caused by geometrical effects: the depth-dependent rates are originally obtained for a plane-parallel cloud, whereas the central star irradiates the disk obliquely. For $50 \%$ of the photons which are scattered in the disk surface, the grazing angle between the disk and the ray path becomes larger than the initial value, which causes deeper penetration into the disk.
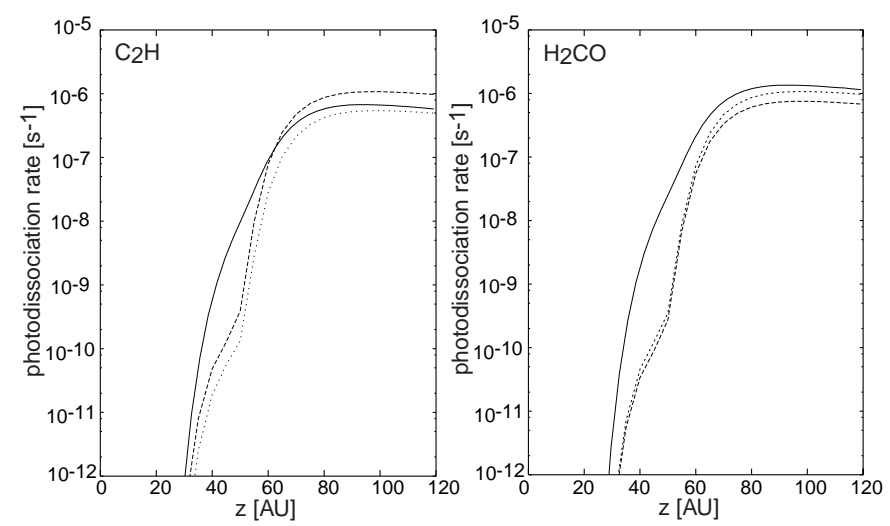

Fig. 3. Photodissociation rates of $\mathrm{C}_{2} \mathrm{H}$ and $\mathrm{H}_{2} \mathrm{CO}$ as functions of height from the midplane at $R=105 \mathrm{AU}$. Rates are calculated using the radiation field obtained via the full 2D Monte Carlo radiative transfer (solid lines). For comparison, rates obtained via the approximate 2D method adopting the depth-dependent function of van Dishoeck (1988) (dotted lines) and the new standard model (dashed lines) are shown.

\subsection{Vertical distribution of molecules}

Using the photodissociation rates calculated in Sect. 3.1, the abundances of the molecules are calculated as a function of time. Figure 4 shows the vertical distribution of the molecules at a disk radius of $R=105 \mathrm{AU}$ at $1 \times 10^{6} \mathrm{yr}$. The dissociation rates in panels $\mathrm{a}$ and $\mathrm{b}$ are obtained via the depth-dependent function (Eq. (3)), where the same model is used as that presented in Paper I, but plotted at a different radius. This model is called the A-2D (approximate 2D) model hereafter. Two improvements are made in this paper: photodissociation rates are calculated via the full 2D radiative transfer, and $\mathrm{H}_{2}$ and $\mathrm{CO}$ dissociation via stellar UV is included. To disentangle the two effects, two models are run. In model ND-2D (no-dissociation, panels $\mathrm{c}$ and $\mathrm{d}$ ), the dissociation rates are obtained via the 


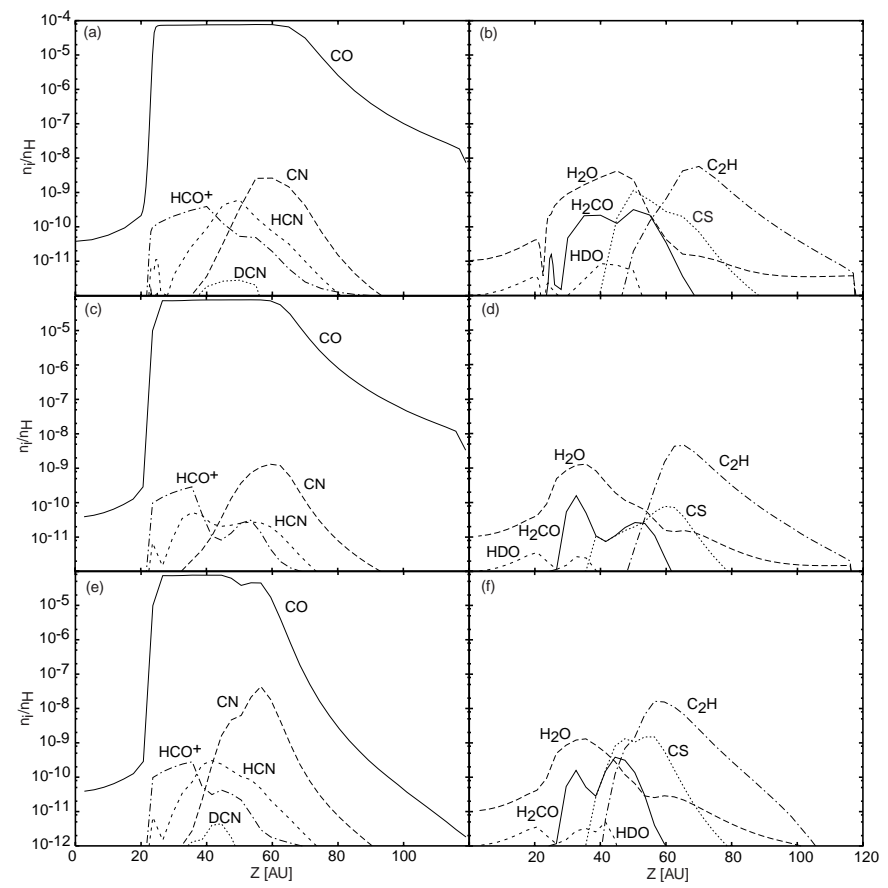

Fig. 4. Vertical distribution of molecules at $R=105 \mathrm{AU}$ and $t=$ $1 \times 10^{6} \mathrm{yr}$. In panels a) and b), the approximate-2D (A-2D) method is adopted with the depth-dependent dissociation rates. The dissociation rates used in panels c) and d) are determined using the full 2D radiative transfer (ND-2D). In both cases the stellar UV is assumed not to be energetic enough to dissociate $\mathrm{H}_{2}$ and $\mathrm{CO}$. In panels e) and f), dissociation rates are determined using the full $2 \mathrm{D}$ radiative transfer, and dissociation of $\mathrm{H}_{2}$ and $\mathrm{CO}$ via the stellar $\mathrm{UV}$ is considered.

full-2D radiative transfer results, but $\mathrm{CO}$ and $\mathrm{H}_{2}$ are not dissociated by the stellar light. Comparison with A-2D (panels a and b) shows that the $\mathrm{CO}$ abundances change only slightly, caused by the scattering and further penetration of interstellar UV photons in full 2D. Most other species have lower abundances because the dissociation rate is enhanced. The results obtained using the full $2 \mathrm{D}$ radiative transfer and $\mathrm{H}_{2}$ and $\mathrm{CO}$ dissociation via the stellar UV are presented in panels e and $\mathrm{f}$. $\mathrm{CO}$ is dissociated even at $Z \sim 50 \mathrm{AU}$ in the $2 \mathrm{D}$ model, while it is largely shielded from UV at $Z \lesssim 60$ AU in the other two cases. The peak abundances of radicals such as $\mathrm{CN}$ and $\mathrm{C}_{2} \mathrm{H}$ (at $Z \sim 60 \mathrm{AU}$ ), are enhanced, because the stellar UV dissociates $\mathrm{CO}$ to provide more atomic carbon in the gas-phase reaction network. This zone corresponds to the so-called "radical zone" found in the chemical networks of PDRs (e.g., Jansen et al. 1995a, 1995b; Sternberg \& Dalgarno 1995). The other species benefit from the $\mathrm{CO}$ dissociation as well; their abundances are higher than in the ND-2D model.

\subsection{Column densities}

The column densities obtained at each radius by integrating the vertical distribution of molecules (Fig. 4) are presented in Fig. 5. The column densities of radicals $\left(\mathrm{CN}\right.$ and $\left.\mathrm{C}_{2} \mathrm{H}\right)$ depend sensitively on the photodissociation treatment, especially at the inner radii. The column densities of $\mathrm{CN}$ and $\mathrm{C}_{2} \mathrm{H}$ in the $\mathrm{A}-2 \mathrm{D}$ and ND-2D models increase with radius because of the lower

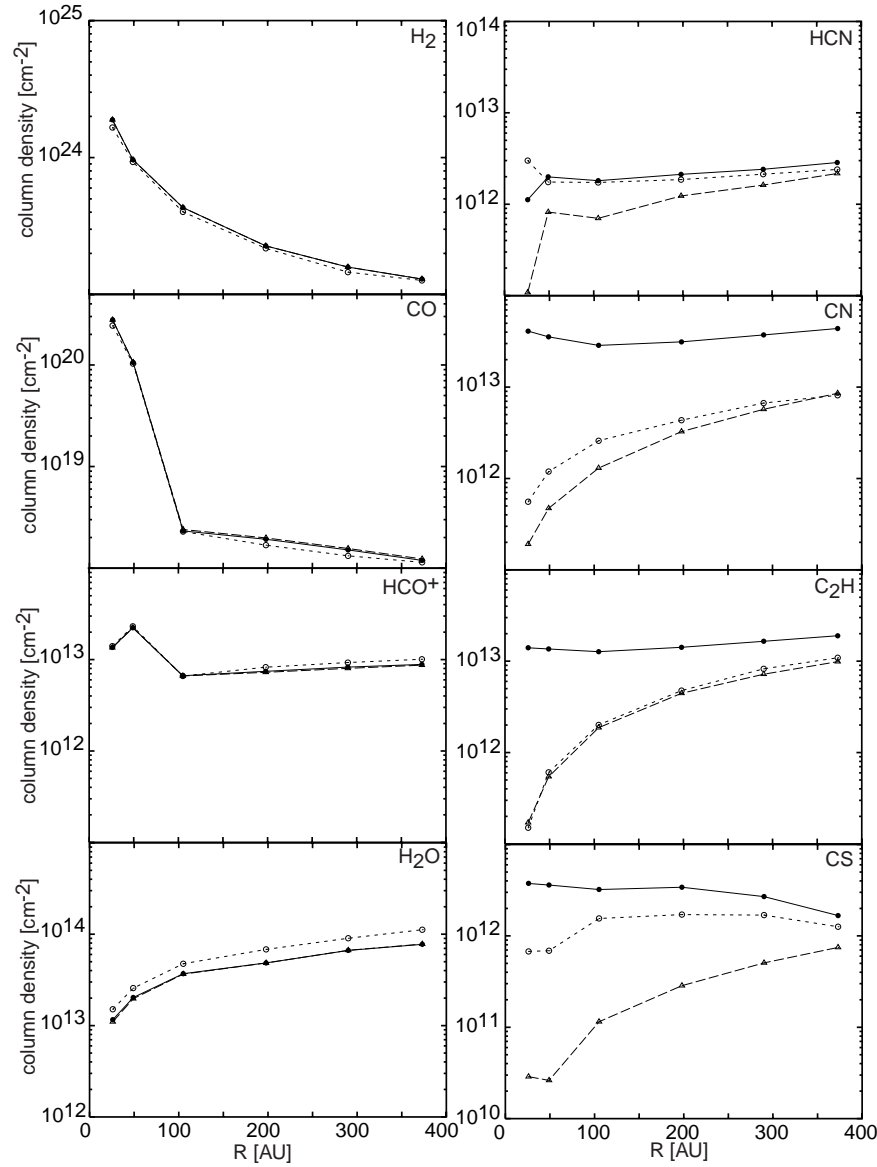

Fig. 5. Radial distribution of column densities at $t=1 \times 10^{6} \mathrm{yr}$. The dashed lines with open circles refer to the A-2D model, the longdashed lines with the solid triangles to the ND-2D model, and the solid lines with closed circles are for the full 2D model.

density and lower flux of the destructive stellar UV in the outer regions. Inclusion of $\mathrm{CO}$ dissociation provides more carbon in the gas-phase reaction network, which significantly enhances the abundances of $\mathrm{CN}$ and $\mathrm{C}_{2} \mathrm{H}$. At the inner radii, the higher dissociation rates of these radicals are compensated by a larger carbon supply. It suggests that an accurate calculation of the radiative transfer of the stellar UV is important when considering the abundances of radical molecules in disks.

Photodissociation of CO via the stellar UV at the disk surface, however, does not affect the column density of CO significantly. It should be noted that Fig. 4 shows the molecular abundances relative to hydrogen nuclei, and that the absolute number density of $\mathrm{CO}$ is highest in the layer with $Z \sim 30$, which contributes predominantly to the $\mathrm{CO}$ column density. Overall, column densities of other species do not significantly depend on the treatment of stellar UV. Close to the star, where the effect of the stellar UV field is largest, differences can be seen. The HCN abundance close to the star shows that $2 \mathrm{D} \mathrm{UV}$ transfer is important.

As argued in Paper I and as can be seen in Fig. 4, molecules are abundant only in layers with certain physical conditions. Since the mass contained in those layers does not vary much with radius, column densities of molecules such as HCN and $\mathrm{H}_{2} \mathrm{CO}$ show little dependence on radius. The column densities 
of $\mathrm{CO}$ and $\mathrm{HCO}^{+}$abruptly change at $R \sim 100 \mathrm{AU}$, inside of which the temperature in the midplane is higher than the sublimation temperature of $\mathrm{CO}(\sim 20 \mathrm{~K})$.

Molecular column densities were also calculated for disks with higher and lower accretion rates of $10^{-7}$ and $10^{-9} M_{\odot} \mathrm{yr}^{-1}$ and correspondingly larger and smaller disk masses. For the same reasons stated above, the column densities are similar to those in Fig. 5, except for $\mathrm{CO}$ and $\mathrm{HCO}^{+}$in the inner radius $(R \lesssim 100 \mathrm{AU})$ and $\mathrm{H}_{2}$, whose column densities are higher (lower) in more (less) massive disks (Paper I).

\subsection{Dependence on the stellar spectrum}

So far, the UV spectrum from the central star has been assumed to be the same as that of the interstellar radiation field, but with an intensity $10^{4}$ times higher than the interstellar field at $R=100$ AU (hereafter referred to as Spectrum A). This spectrum was motivated by Herbig \& Goodrich (1986) comparing IUE data in the wavelength range between 1450 and $1850 \AA$ towards different $\mathrm{T}$ Tauri stars. In this subsection, the sensitivity of our results to the UV spectrum of the central star is investigated by calculating two other models with different stellar spectra: the observed spectrum of the T Tauri star TW Hya (Spectrum B), and a black body spectrum at $T_{*}=4000 \mathrm{~K}$ without any excess UV (Spectrum C). Costa et al. (2000) fitted the UV continuum from TW Hya, observed with the IUE satellite, with a sum of free-free plus free-bound emission at $3 \times 10^{4} \mathrm{~K}$, plus a $4000 \mathrm{~K}$ black body spectrum for the star and a black body emission at $7900 \mathrm{~K}$ covering $5 \%$ of the stellar surface. We adopt his numerical data at the stellar surface as Spectrum B. Note that no observational constraints are available below $1200 \AA$, especially in the critical 912-1100 A range where $\mathrm{H}_{2}$ and $\mathrm{CO}$ are photodissociated. It will be important to obtain such data in the future with the FUSE satellite. The third case with Spectrum C gives a lower limit to the UV radiation from the central star, and may correspond to the situation for disks around weak-line T Tauri stars (WTTS), although the

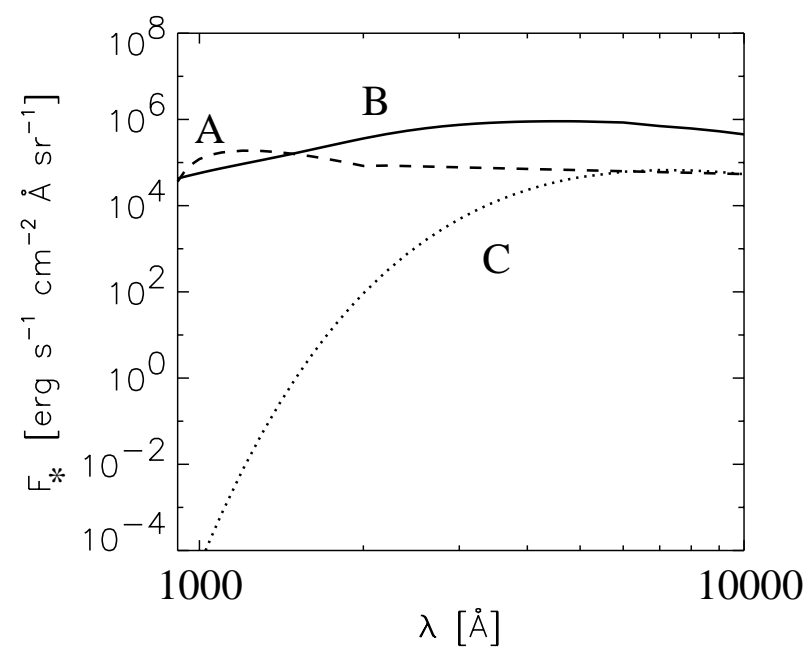

Fig. 6. Comparison of the mean intensity at a radius of $R=105 \mathrm{AU}$ for the three stellar spectra: Spectrum A (dashed line), Spectrum B (solid line), and Spectrum C (dotted line) (see text). physical structure of such disks would be different from those around classical T Tauri stars. As in the full 2D model of the previous section, the photodissociation rates are obtained via full $2 \mathrm{D}$ radiation transfer, and dissociation of $\mathrm{H}_{2}$ and $\mathrm{CO}$ via stellar UV is taken into account. Photodissociation by the interstellar radiation is included in all models. Figure 6 shows the spectrum at the disk surface at $R \approx 100 \mathrm{AU}$ for the three stellar models.

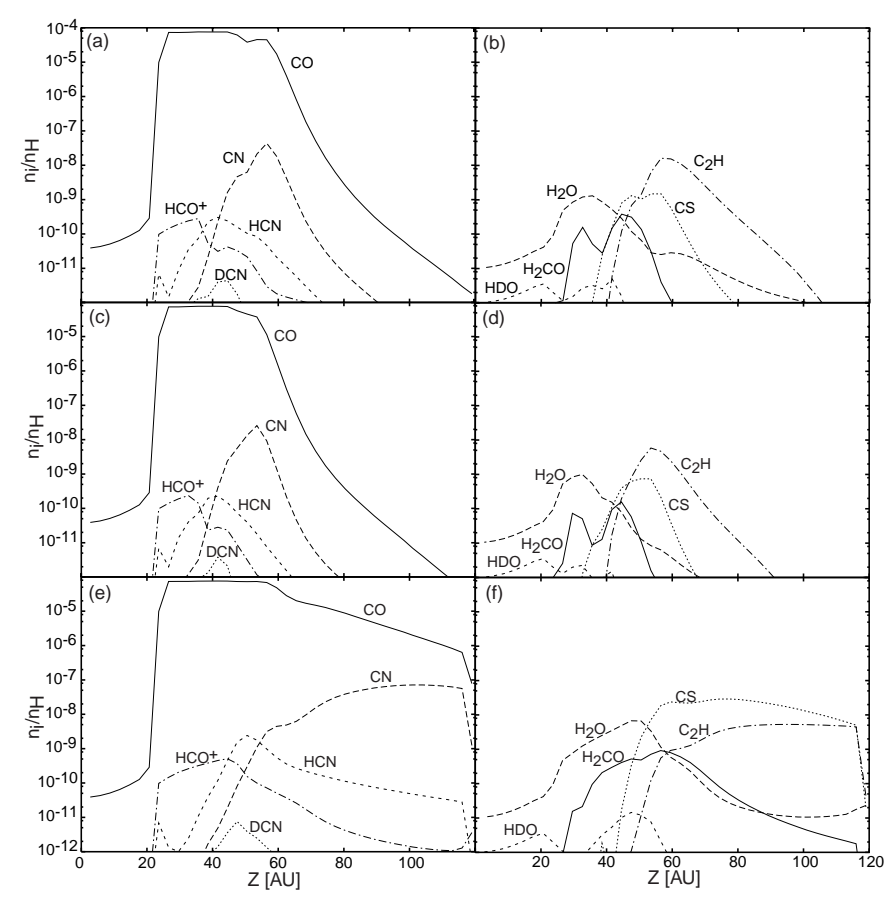

Fig. 7. Vertical distribution of molecules at $R=105 \mathrm{AU}$ and $t=1 \times$ $10^{6}$ yr. Panels a) and b) are for Spectrum A, panels c) and d) are for Spectrum B and panels e) and f) for Spectrum C.

Figures 7 and 8 show the vertical distribution of molecules at $R=105 \mathrm{AU}$, and the radial distribution of column densities for the three models with different stellar UV spectra, respectively. The model using Spectrum B shows almost the same distributions and column densities as those obtained with Spectrum A. This is not surprising since the spectrum of the interstellar radiation field has a color temperature of $3 \times 10^{4} \mathrm{~K}$, close to that of the free-free and free-bound component in Spectrum B. In the model without excess UV (Spectrum C), on the other hand, the molecular distributions are far more extended toward the surface layers. The lower photodissociation rates at the disk surface tend to enhance the column densities, but the radical $\left(\mathrm{CN}\right.$ and $\left.\mathrm{C}_{2} \mathrm{H}\right)$ column densities in the inner regions are lowered because of the smaller amount of atomic carbon supplied via CO dissociation in the intermediate layers.

\section{Molecular line emission}

\subsection{Comparison of models}

The comparison of our new results obtained via the full-2D radiation transfer with the A-2D model and the ND-2D model 


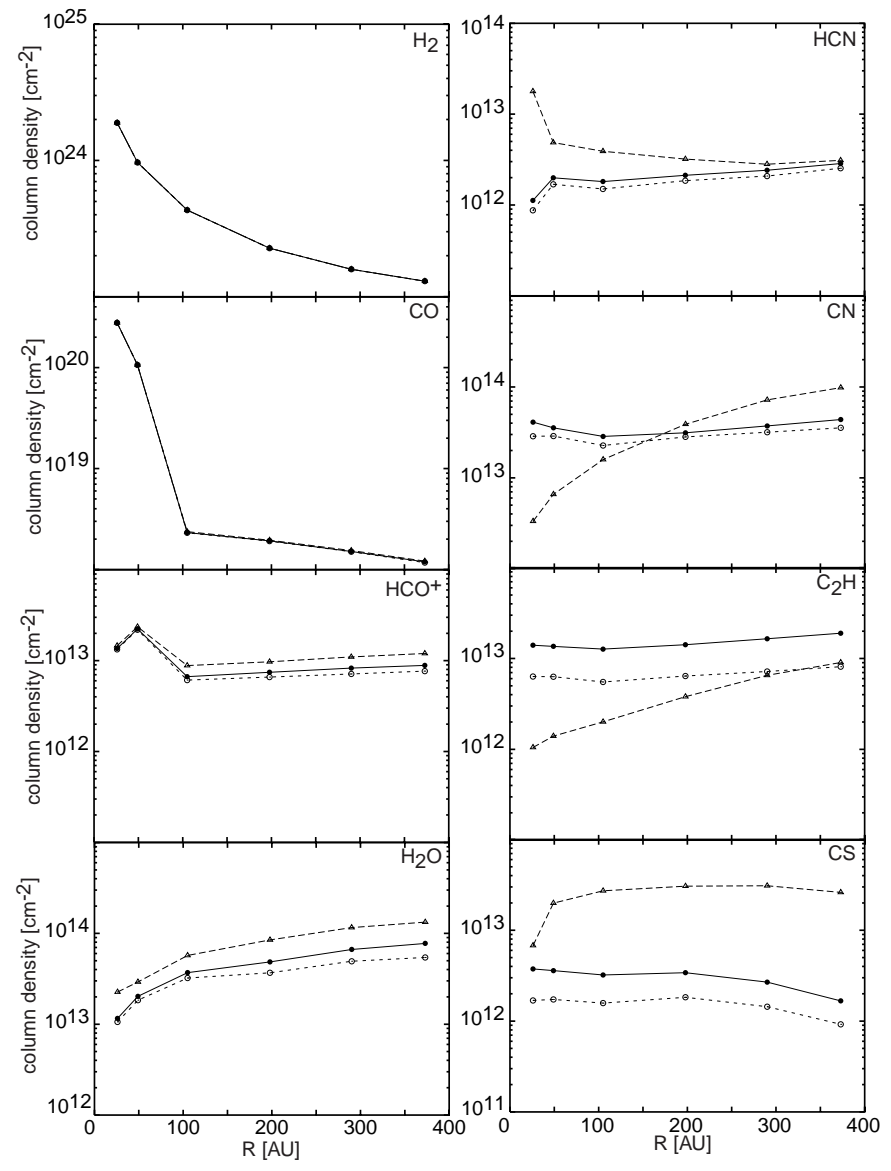

Fig. 8. Radial distribution of molecular column densities at $t=1 \times$ $10^{6} \mathrm{yr}$. Solid lines with closed circles are for Spectrum A, dashed lines with open circles for Spectrum B, and long-dashed lines with triangles for Spectrum C.

shows some differences in molecular distributions, especially for radicals.

The question is if these differences are significant with the present day observations or whether any differences are minimized after solving the radiative transfer in the millimeter lines and convolving the data with the telescope beam. The CO and $\mathrm{HCO}^{+}$(sub-)millimeter lines are extremely optically thick in these models. To get more information on the emission from deeper layers in the disk, the radiative transfer has been run for ${ }^{13} \mathrm{CO}$ and $\mathrm{H}^{13} \mathrm{CO}^{+}$as well. These species are assumed to follow the abundances of $\mathrm{CO}$ and $\mathrm{HCO}^{+}$respectively, but their abundances are lowered by a factor of 60 , similar to the $\left[{ }^{12} \mathrm{C}\right] /\left[{ }^{13} \mathrm{C}\right]$ isotopic ratio in the solar neighborhood. This overestimates the ${ }^{13} \mathrm{CO}$ abundance for the full-2D case since $\mathrm{CO}$ is more selfshielding compared to ${ }^{13} \mathrm{CO}$.

As in Paper I, the level populations and emission lines of different species are calculated using a 2D line radiative transfer Monte Carlo code (Hogerheijde \& van der Tak 2000). The calculations assume that the $400 \mathrm{AU}$ radius disk is seen at an inclination of $60^{\circ}$ at a distance of $150 \mathrm{pc}$, appropriate for the case of $\mathrm{LkCa} 15$. The results are presented in two ways; first, the emission profiles for a few characteristic molecules are plotted, and second, the ratios of integrated intensities of the lines are compared in several tables. In Fig. 9, lines are shown for
Table 1. The ratios of the integrated intensities for the full-2D, ND-2D and A-2D models (see text). The line emissions have been convolved with a beam equal to the size of the disk on the sky (5.33").

\begin{tabular}{lcrcc}
\hline \hline Molecule & line & $v[\mathrm{GHz}]$ & full-2D & ND-2D \\
\cline { 5 - 6 } \cline { 5 - 5 } & & & $\mathrm{A}-2 \mathrm{D}$ & $\mathrm{A}-2 \mathrm{D}$ \\
\hline $\mathrm{CO}$ & $6-5$ & 691.473 & 0.88 & 0.97 \\
$\mathrm{CO}$ & $3-2$ & 345.796 & 0.92 & 0.98 \\
$\mathrm{CO}$ & $2-1$ & 230.538 & 0.93 & 0.98 \\
${ }^{13} \mathrm{CO}$ & $3-2$ & 330.587 & 0.98 & 1.03 \\
${ }^{13} \mathrm{CO}$ & $1-0$ & 220.399 & 1.05 & 1.08 \\
$\mathrm{HCO}^{+}$ & $4-3$ & 356.734 & 0.94 & 0.93 \\
$\mathrm{HCO}^{+}$ & $1-0$ & 89.189 & 0.91 & 0.89 \\
$\mathrm{H}^{13} \mathrm{CO}^{+}$ & $4-3$ & 346.999 & 0.94 & 0.93 \\
$\mathrm{H}^{13} \mathrm{CO}^{+}$ & $1-0$ & 86.754 & 0.98 & 0.96 \\
$\mathrm{CN}^{\mathrm{CN}}$ & $3-2$ & 340.248 & 10.1 & 0.75 \\
$\mathrm{HCN}$ & $2-1$ & 226.875 & 5.81 & 0.85 \\
$\mathrm{HCN}$ & $4-3$ & 354.506 & 1.21 & 0.81 \\
\hline
\end{tabular}

$\mathrm{CO} 3-2, \mathrm{HCO}^{+} 4-3, \mathrm{CN}_{3}-2$ and $\mathrm{HCN} 4-3$. The CN 3-2 line is modeled assuming that the three fine-structure lines are well shifted from each other, with each fine-structure line a combination of three unresolved hyperfine components. Therefore, the CN 3-2 line shows a regular double-peaked profile instead of a combination of three double-peaked structures summed at slightly shifted velocities.
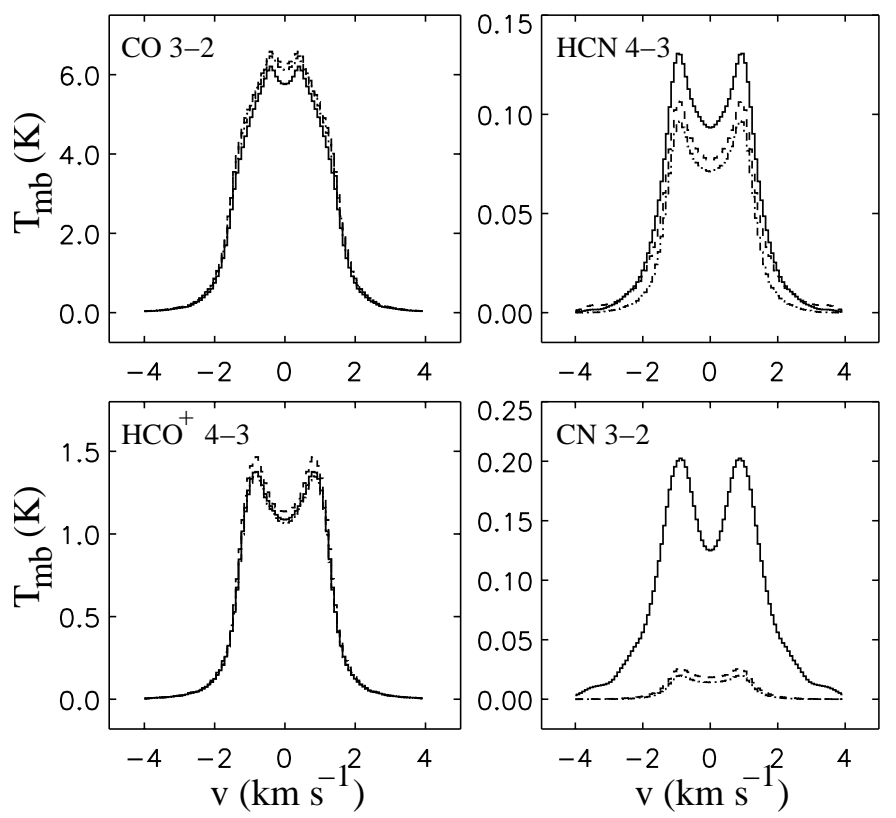

Fig. 9. Comparison of emission lines for the full-2D radiative transfer model (solid lines), the $2 \mathrm{D}$ radiative transfer model without $\mathrm{CO}$ and $\mathrm{H}_{2}$ dissociation (ND-2D; dot-dashed) and the approximate 2D model (A-2D; dashed lines). The CO 3-2, $\mathrm{HCO}^{+} 4-3, \mathrm{CN} 3-2$ and $\mathrm{HCN} 4-3$ lines are convolved with a beam equal to the size of the source on the $\operatorname{sky}\left(5.33^{\prime \prime}\right)$.

In Table 1, the ratios of the integrated intensities of the full$2 \mathrm{D}$ and $\mathrm{A}-2 \mathrm{D}$ as well as the ND-2D over A-2D models are given for a few lines. All models refer to a beam size equal to 
Table 2. Integrated intensities for the three stellar spectra (A, B and C) convolved with a beam of 5.33".

\begin{tabular}{lcccc}
\hline \hline molecular line & $\begin{array}{c}\mathrm{A} \\
{\left[\mathrm{K} \mathrm{km} \mathrm{s}^{-1}\right]}\end{array}$ & $\begin{array}{c}\mathrm{B} \\
{\left[\mathrm{K} \mathrm{km} \mathrm{s}^{-1}\right]}\end{array}$ & $\begin{array}{c}\mathrm{C} \\
{\left[\mathrm{K} \mathrm{km} \mathrm{s}^{-1}\right]}\end{array}$ \\
\hline $\mathrm{CO}$ & $6-5$ & 11.19 & 11.79 & 12.65 \\
$\mathrm{CO}$ & $3-2$ & 16.92 & 17.62 & 18.74 \\
$\mathrm{CO}$ & $2-1$ & 17.86 & 18.52 & 19.58 \\
${ }^{13} \mathrm{CO}$ & $3-2$ & 7.93 & 8.05 & 8.28 \\
${ }^{13} \mathrm{CO}$ & $2-1$ & 7.84 & 7.93 & 8.09 \\
${ }^{13} \mathrm{CO}$ & $1-0$ & 5.28 & 5.31 & 5.38 \\
$\mathrm{HCO}^{+}$ & $4-3$ & 3.38 & 3.72 & 4.45 \\
$\mathrm{HCO}^{+}$ & $1-0$ & 2.07 & 2.31 & 2.93 \\
$\mathrm{H}^{13} \mathrm{CO}^{+}$ & $4-3$ & 0.10 & 0.11 & 0.14 \\
$\mathrm{H}^{13} \mathrm{CO}^{+}$ & $1-0$ & 0.04 & 0.05 & 0.06 \\
$\mathrm{CN}^{\mathrm{CN}}$ & $3-2$ & 0.68 & 0.66 & 0.15 \\
$\mathrm{CNN}^{\mathrm{HCN}}$ & $2-1$ & 1.94 & 2.00 & 1.01 \\
$\mathrm{HCN}$ & $4-3$ & 0.35 & 0.37 & 0.46 \\
\hline
\end{tabular}

the apparent size of the disk at a distance of $150 \mathrm{pc}$. In both Fig. 9 and Table 1 the CO emission is slightly lowered in the full-2D case due to the dissociation by stellar UV.

Due to the dissociation of $\mathrm{CO}$ leading to atomic carbon, the $\mathrm{CN}$ abundance and emission is enhanced up to an order of magnitude. This is clearly seen in Fig. 9, where the A-2D and ND-2D models have similar emission profiles, but full-2D is much higher. $\mathrm{HCN}$ is linked to $\mathrm{CN}$ and its abundance is enhanced as well; however, in contrast with $\mathrm{CN}$, which can only be dissociated at wavelengths less than $1000 \AA$, HCN can be easily destroyed again by photodissociation, resulting in both the abundances and line emission to rise only mildly. Among the three models, the ND-2D model gives the weakest HCN emission.

\subsection{Dependence on stellar radiation field}

In the previous section it was shown that the chemical abundances need a good description of the mean intensity of the UV radiation field. The column densities of radicals such as $\mathrm{CN}$ are especially sensitive to the UV spectrum. The models with excess UV radiation (Spectrum A and B) have a flat column density distribution throughout the disk, while the model without excess UV (Spectrum C) has an increasing CN column density toward the outer radius. Figure 10 shows line profiles of $\mathrm{HCN}, \mathrm{HCO}^{+}$and $\mathrm{CN}$ for the three different stellar spectra. The lines of $\mathrm{HCO}^{+}$nicely reflect the column densities shown in Fig. 8; the model with Spectrum C gives the strongest emission. The HCN column densities have similar values at larger radii, which is reflected in the 4-3 emission lines. At smaller radii the column of the model with Spectrum $\mathrm{C}$ dominates by a factor of 5 . This is reflected in the higher emission in the wings of the line profile. The excess emission at $|v| \sim 4 \mathrm{~km} \mathrm{~s}^{-1}$ is due to the undersampled grid in the inner region, but this does not effect the integrated emission. The $\mathrm{CN}$ emission is discussed in more detail in Sect. 4.3.

For a broader overview of the calculated emission lines, the integrated intensities are given in Table 2 for several species and lines. These results were again calculated for the source seen with a beam of 5.33". The CO emission lines show only a small dependence on the stellar UV field $(\approx 10 \%)$, where the case without excess UV (Specrum C) shows the highest integrated flux. The ${ }^{13} \mathrm{CO}$ lines show an even smaller difference since most of the emission comes from the warm intermediate layers and is not affected by dissociation in the surface layer. $\mathrm{HCO}^{+}$and its main isotopomer $\mathrm{H}^{13} \mathrm{CO}^{+}$have a similar behavior, but with a larger difference $(\approx 30-50 \%)$. The $\mathrm{HCO}^{+}$emission is strongest for the star with no excess UV (Spectrum C), and is slightly stronger for Spectrum B than A.

\subsection{NLTE effects on the CN line emission.}

Special attention should be paid to the $\mathrm{CN}$ lines, because they are among the strongest lines observed in several disks, and because LTE excitation is not a good approximation for this molecule as shown below. The integrated intensities of the $\mathrm{CN}$ lines are larger by a factor of 2 to 3 (Table 2) in the models with excess UV (Spectrum A and B), even though the CN column densities are a factor of two higher in the outer regions of the disk for the model without excess UV (Spectrum C) (Fig. 8). This appears counterintuitive since the outer disk, due to its larger surface, should dominate the line emission. This is not the case since most of the additional $\mathrm{CN}$ in the model with Spectrum $C$ is located in the upper most layers of the disk where the density is so low that the levels become subthermally excited. To show the effect of the NLTE excitation, the profiles of the CN 3-2 line for the three stellar spectra are plotted for NLTE and LTE populations in Fig. 11. In the LTE case the emission from the model with Spectrum $\mathrm{C}$ dominates the lines from Spectrum A and B as expected when considering the column densities (Fig. 8). For the NLTE calculations, the order of the emission reverses since most of the $\mathrm{CN}$ column in the model with Spectrum C is at low density.

In addition, a few of the hyperfine lines of the $J=1-0$ transition experience small masing effects due to a population inversion. This makes NLTE excitation effects important for all $\mathrm{CN}$ levels in these disk environments. The modeling and interpretation of any of the $\mathrm{CN}$ levels should therefore be done with a detailed radiative transfer study.

\subsection{High resolution simulation of $\mathrm{CN} / \mathrm{HCN}$}

The CN and HCN column densities (Fig. 8) show clear differences for the different radiation fields. Their abundance ratio in the disk is a sensitive function of radius due to the different radial gradients of the column densities of $\mathrm{CN}$ and $\mathrm{HCN}$. In the future, telescopes like the Atacama Large Millimeter Array (ALMA) will be able to probe this ratio spatially. In order to simulate ALMA observations, the molecular line intensities are calculated assuming a FWHM Gaussian beam of $0.3^{\prime \prime}$, which is chosen based on the spatial resolution of the model grid and which will easily be reached with ALMA. In Fig. 12c the velocity integrated intensity ratio along the major axis of the disk is plotted for spectra B and C. The intensity ratio for spectrum B peaks close to the star and drops to a 15 times lower 

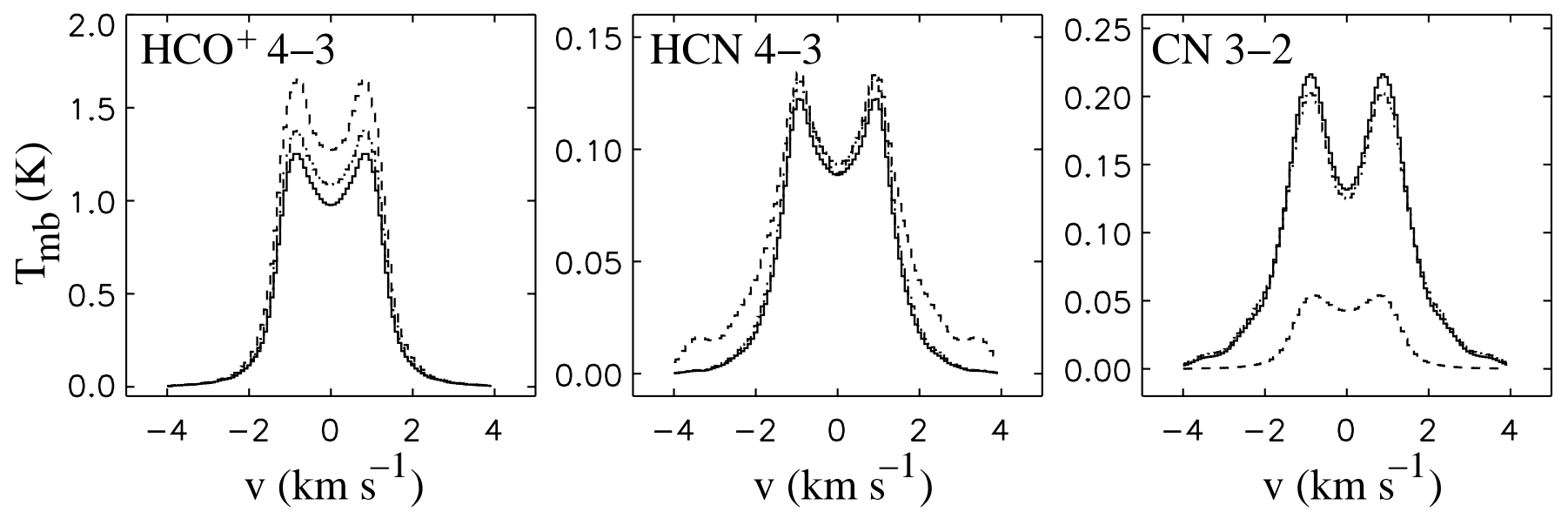

Fig. 10. Comparison of $\mathrm{HCO}^{+} 4-3$ (left), $\mathrm{HCN}$ 4-3 (middle) and CN 3-2 (right) emission lines for the three stellar spectra. The dash-dotted lines are for Spectrum A, the solid lines for Spectrum B, and the dashed lines for Spectrum C. The spectra have been convolved with a beam of $5.33^{\prime \prime}$.
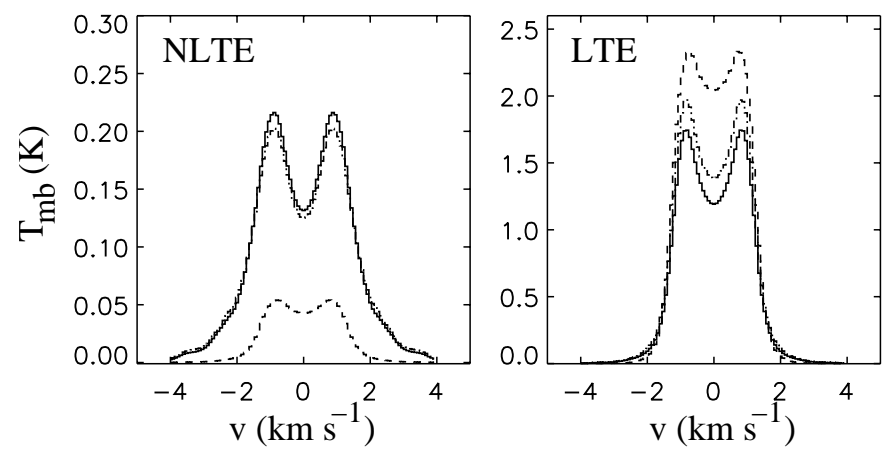

Fig. 11. Comparison of the CN 3-2 emission line for NLTE (left) and LTE (right) populations. The dash-dotted lines are for Spectrum A, the solid lines for Spectrum B, and the dashed lines for Spectrum C. The spectra have been convolved with a beam of 5.33".

ratio at large radii. Spectrum $\mathrm{C}$ shows the opposite behavior rising by a factor 4 from close to the star to larger radii. The emissions at these large radii are similar (Figs. 12b, c), so that high resolution is essential for distinguishing the effects of different UV radiation fields. For a better understanding of the emission profile, the beam averaged column densities and integrated intensities for $\mathrm{CN}$ and $\mathrm{HCN}$ are given in Figs. 12a and b, respectively. The drop of intensities at $\sim 2.5^{\prime \prime}$ is due to the partial filling of the beam. In both cases the ratio of the intensities for spectrum B and C is smaller than the ratio of the column densities by roughly an order of magnitude; in the inner most region with spectrum $\mathrm{C}$, for example, the column density ratio of $\mathrm{CN}$ to $\mathrm{HCN}$ is almost unity, while the intensity ratio is $\lesssim 0.1$. The expected sensitivity of ALMA at $345 \mathrm{GHz}$ in a $0.3^{\prime \prime}$ beam is $\sim 0.3 \mathrm{~K} \mathrm{~km} \mathrm{~s}^{-1}$ in $1 \mathrm{hr}$. Thus, the $\mathrm{HCN}$ and $\mathrm{CN}$ distribution in the inner $0.6^{\prime \prime}$ can readily be imaged and the Spectrum B and C models should be easily distinguishable. Imaging of $\mathrm{CN}$ in the outer part of the disk will require longer integration times.

\section{Discussion}

The calculated abundances and column densities show orders of magnitude variations between different models. The

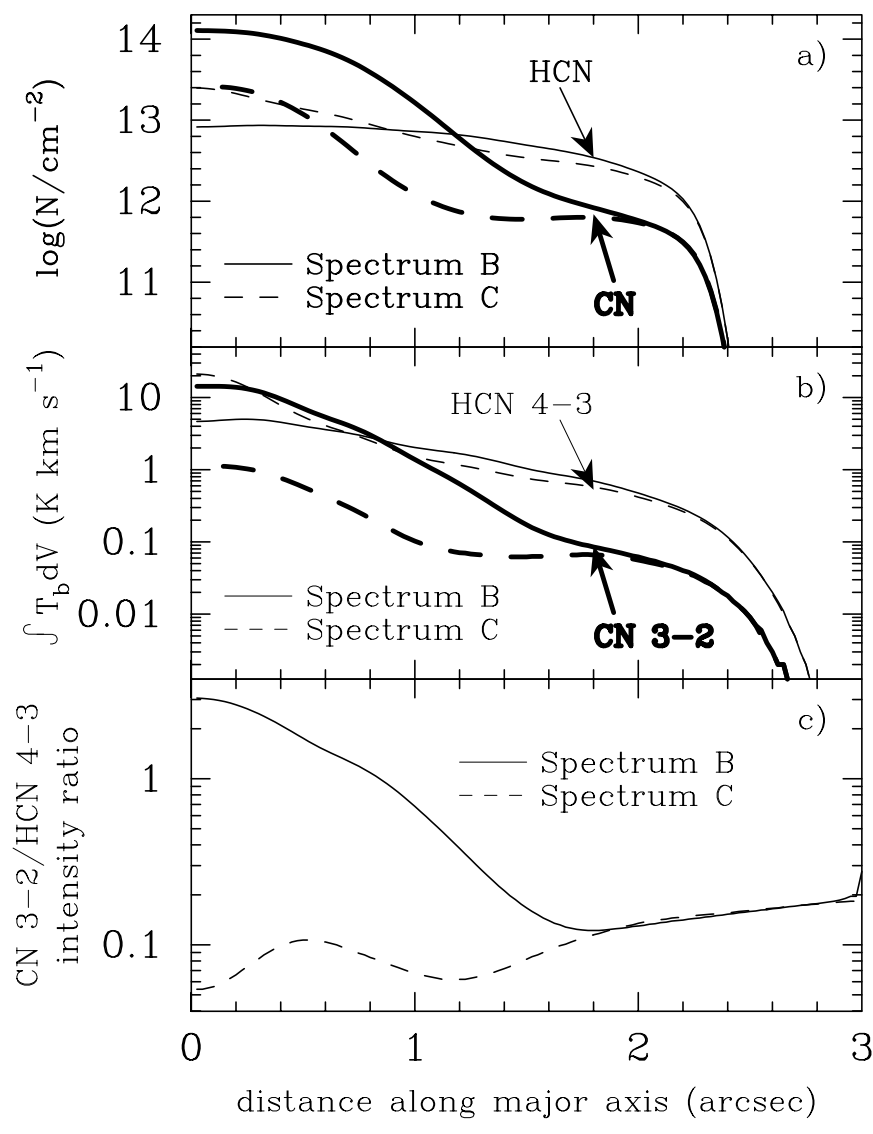

Fig. 12. a) The beam averaged column densities of $\mathrm{CN}$ and $\mathrm{HCN}$. b) Integrated intensities for the $\mathrm{CN} 3-2$ and $\mathrm{HCN} \mathrm{4-3}$ transition. c) Ratio of the integrated intensity of $\mathrm{CN} 3-2 / \mathrm{HCN} 4-3$. The modeled intensities and columns were calculated using a beam of $0.3^{\prime \prime}$. The solid lines represents the emission of a star with stellar UV (Spectrum B), the dashed lines a T Tauri star with no excess UV (Spectrum C). The thick lines in the plots a) and b) represent the $\mathrm{CN}$ and the thin lines the HCN column-densities and intensities.

inclusion of ND-2D UV radiative transfer instead of an approximate $2 \mathrm{D}$ transfer decreases the abundances of species like $\mathrm{HCN}, \mathrm{CN}$ and $\mathrm{CS}$. When $\mathrm{CO}$ dissociation via stellar UV is 
taken into account, a large amount of atomic carbon is produced, which is converted to radicals $\left(\mathrm{CN}, \mathrm{C}_{2} \mathrm{H}\right)$ and direct reaction products (CS, HCN). The adopted stellar spectrum has a clear effect on the column densities of most species except for CO. Especially the $\mathrm{CN}$ and $\mathrm{HCN}$ column densities show different slopes with increasing radius. These variations should be detectable with sufficient spatial resolution as modeled in Fig. 12.

The effect of UV radiative transfer and the stellar UV spectrum are less clear in the integrated line intensities with larger beams for the following reasons. First, the telescope beams available, now and in the near future, average the disk over the entire beam. Second, most lines observed to date are the brightest lines available. A large number of these are optically thick; $\mathrm{CO}$ for instance is only capable of probing the upper layers of the disk. Third, some lines suffer from sub-thermal excitation. The NLTE effects can be severe in the optically thin upper layers of the disk, while the LTE intermediate layers are at a large optical depth.

A comparison between different millimeter line radiative transfer codes (van Zadelhoff et al. 2002) shows that the standard deviation on the level populations can reach $12 \%$ for the higher levels in NLTE. These calculations were performed for an extreme combination of optical depth $(\tau)$ and NLTE. Even though the $\tau$ in disks is very high, the levels are not as far from LTE as in the above mentioned calculation. It is therefore safe to assume that the molecular line calculation in this paper (Figs. 9 and 10) has "error-bars" similar or less than 12\%. This is smaller than the error-bars on the observations, which are estimated to be roughly $20 \%$.

In Paper I, the model results underestimated the observed molecular abundances of species such as $\mathrm{CN}$ and $\mathrm{HCN}$, and overestimates $\mathrm{CO}$ in most disks. One of the motivations of the present study is to check if the inclusion of $\mathrm{CO}$ dissociation via stellar UV could resolve these discrepancies. In comparison to the results presented in Paper I, both the $\mathrm{CN}$ emission and the $\mathrm{CN} / \mathrm{HCN}$ ratio has significantly increased using the full2D UV radiative transfer code. The column density of $\mathrm{C}_{2} \mathrm{H}$ is also increased. However, the $\mathrm{CO}$ column density itself is not much affected by dissociation via the stellar UV. The column densities of non-radical species such as $\mathrm{HCN}$ are almost the same as in Paper I and are thus lower than observed in some sources.

There are several important physical processes which need to be investigated in future studies (see also Sect. 2.1). The UV transfer was calculated here using a particular set of grain scattering properties. The mean intensities derived in the disk change when different properties are adopted. Since the abundances do not depend linearly on the mean intensity, they are not expected to differ much as long as the gas/dust ratio and grains sizes are similar to those in interstellar clouds. If the dust sizes have increased to mm-sized grains, this would alter both the dust properties and the dust settling. This may allow more UV photons to reach deeper into the disk, enhancing the dissociation of $\mathrm{CO}$.

The inclusion of X-rays could significantly change the chemical abundances (Aikawa \& Herbst 1999, 2001). The secondary UV field generated by the excited $\mathrm{H}_{2}$ could result in a higher abundance of radicals at lower disk heights. This would enhance the total line emission due to both the higher $\mathrm{CN}$ and HCN column densities and the increased excitation of the molecules at higher densities. Hydrodynamics such as accretion and turbulent mixing would smooth out molecular stratification to some extent, and transport of radical species would affect the chemical processes in neighboring regions. On the observational side spatially and spectrally resolved observations are needed to find unique tracers of different processes.

\section{Conclusion}

The main conclusions from this work are:

- The transfer of stellar ultraviolet radiation has to be treated in a two-dimensional manner for a correct abundance determination of radicals like $\mathrm{CN}$ and $\mathrm{C}_{2} \mathrm{H}$.

- The photodissociation of $\mathrm{CO}$ by the stellar radiation supplies additional atomic carbon, which leads to significantly higher abundances of molecules, especially of radicals like $\mathrm{CN}$ and $\mathrm{C}_{2} \mathrm{H}$. $\mathrm{CO}$ itself shows no detectable dependence on the stellar radiation field, making it a poor tracer of the chemical processes in disks.

- The abundances of the molecules in the "radical region" are also sensitive to the adopted stellar UV spectrum. This effect will not necessarily be reflected in the current observed line intensities, however, since the lines may be sub-thermally excited in the surface layers where the abundances are most enhanced. Spatially resolved observations by ALMA are needed to distinguish the different scenarios.

- Observational constraints on the fluxes from T Tauri and Herbig Ae stars at ultraviolet wavelengths, in particular in the critical 912-1100 ̊ region, are urgently needed.

Acknowledgements. The authors are grateful to the referee for suggestions and comments that improved the paper considerably. They also thank P. D'Alessio and V. Costa for sending and discussing the disk model and stellar UV field used in the paper. GJvZ acknowledges M. Spaans and C. P. Dullemond for helpful discussions on the UV radiative transfer. Astrochemistry in Leiden is supported by a SPINOZA grant from The Netherlands Organization for Scientific Research (NWO). Y.A. is supported by the Grant-in-Aid for Scientific Research of the Ministry of Education, Culture, Sports, Science and Technology (13011203 and 14740130). The chemical models were carried out at the Astronomical Data Analysis Center of National Astronomical Observatory in Japan.

\section{Appendix A: Axi-symmetric 2D continuum radiative transfer}

A two-dimensional (2D) axi-symmetric Monte Carlo code was set up to calculate the transfer of ultraviolet radiation by absorption and scattering due to grains. In the calculation, the photons are actually followed in 3D, but by applying axial symmetry the grid can be rotated at any time in $\Phi$ such that the photon comes back to the $\Phi=0$ plane. This causes the photons to follow hyperbolic paths in the $2 \mathrm{D} \Phi=0$ plane. The main reason for adopting a $2 \mathrm{D}$ rather than $3 \mathrm{D}$ approach is the increase in accuracy in the solution for the same number of photons or amount of computer time. 


\section{A.1. Photon propagation}

Each photon is started at the source of radiation with an intensity given by the boundary conditions for the source. These boundary conditions are discussed in Sect. A.4. A photon package can undergo two different processes: absorption and scattering by dust. A model photon represents the amount of energy in a wavelength bin, given by the total surface of the source multiplied by the flux through this surface divided by the number of photons in this wavelength bin. Per wavelength bin, one needs sufficient resolution to sample all possible angles of emission. In general the total number of photons per wavelength bin $\delta \lambda$ is given by

$N_{\text {phot }, \delta \lambda}=C 1 \times n \phi \times n \theta \times n R \times n z$,

where $n R$ and $n z$ are the number of cells in the $R-$ and $z$-directions, respectively, and $n \phi$ and $n \theta$ are the directional resolutions. One can multiply this by an arbitrary constant $(\mathrm{C} 1 \geq 1)$ to improve on the statistics. For a reasonably sized grid $(n R=n z=70), 10^{6}$ photons suffice in practice per wavelength bin. In general 34 wavelength bins are used in between $912 \AA$ and $1 \mu \mathrm{m}$.

The initial energy stored in each photon package is given by

$I_{\mathrm{i}}(0, \lambda)=\frac{F_{\lambda} \times S}{N_{\text {phot }, \delta \lambda}}$

where $F_{\lambda}$ is the flux entering the system and $S$ the total surface it passes through.

At the surface of emission source, the scattering optical depth is first calculated using a random number generator

$\tau_{\text {scat }}=-\ln (1-\zeta)$

with $\zeta$ a random number between 0 and 1 . This optical depth can be converted to a total absorption optical depth

$\tau_{\mathrm{abs}}=K_{\lambda} \times \tau_{\text {scat }}$,

where $K_{\lambda}$ is the fraction of absorption compared to scattering. The photon package travels through the grid until it reaches the optical depth $\left(\tau_{a b s}\right)$ at which it scatters. In the mean time, its intensity drops according to

$I_{i}(s+\Delta s, \lambda)=I_{i}(s, \lambda) \mathrm{e}^{-\Delta \tau_{\lambda}}$

where $\Delta \tau_{\lambda}$ is related to the physical properties through

$$
\begin{aligned}
\Delta \tau_{\lambda} & =\frac{1}{2.5 \log _{10}(\mathrm{e})} \times \Delta A_{\lambda} \\
& =\frac{g d(R, z)}{2.5 \log _{10}(\mathrm{e})} \times \frac{\Delta s \times n\left(\mathrm{H}+2 \mathrm{H}_{2}\right)}{1.59 \times 10^{21}} \times \frac{A_{\lambda}}{A_{\mathrm{v}}}
\end{aligned}
$$

with $A_{\lambda}$ the visual extinction at wavelength $\lambda, g d(R, z)$ the gasto-dust ratio compared to $100, \Delta s$ the path length traveled within a grid cell and $n\left(\mathrm{H}+2 \mathrm{H}_{2}\right)$ the total number density in the grid cell. The relation $n(\mathrm{H})+2 n\left(\mathrm{H}_{2}\right)=1.59 \times 10^{21} A_{v}$ is taken from Savage et al. (1977). When the photon package reaches the point of scattering, the entire package changes direction according to the Henyey-Greenstein phase function (HenyeyGreenstein 1941; Witt 1977):

$P\left(\cos \alpha, g_{\lambda}\right)=\frac{1-g_{\lambda}^{2}}{4 \pi\left[1+g_{\lambda}^{2}-2 g_{\lambda} \cos \alpha\right]^{3 / 2}}$

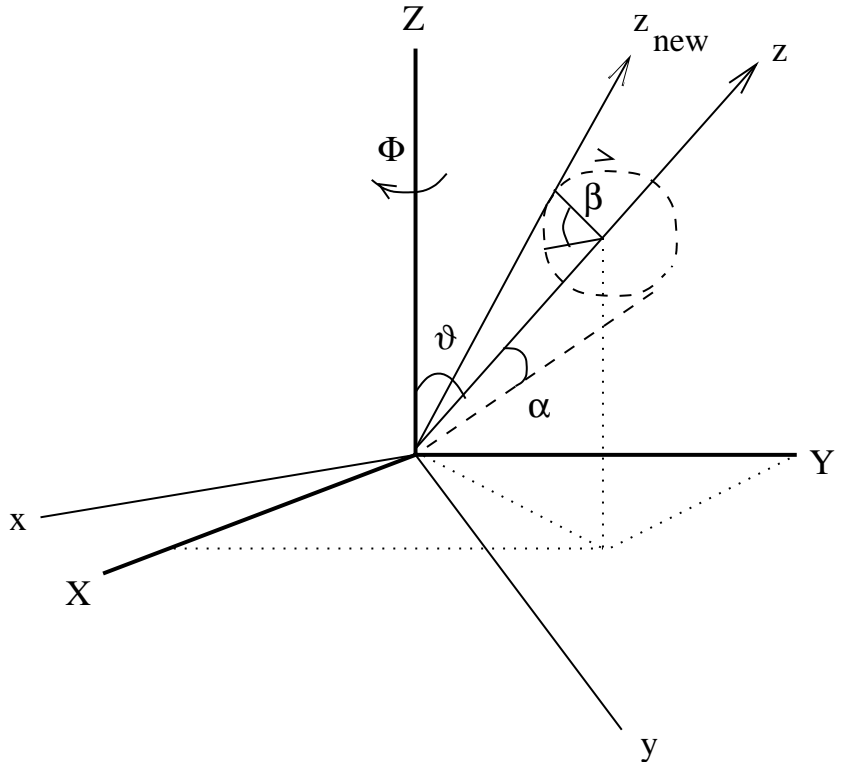

Fig. A.1. Scattering of a photon propagating in the direction $(\phi, \theta)$ through angles $\alpha$ and $\beta$. The new photon direction will lie on a cone defined by $\alpha$ and $\beta$.

with $g_{\lambda}=\langle\cos \alpha\rangle$, the mean scattering angle. The angles under which the photon scatters are in reference to the original direction of motion [defined with an $(x, y, z)$ coordinate system, such that $z$ is in the direction of the photon propagation prior to scattering]

$\alpha=\arccos \left(\frac{1}{2 g_{\lambda}}\left[\left(1+g_{\lambda}^{2}\right)-\left(\frac{1-g_{\lambda}^{2}}{1-g_{\lambda}+2 g_{\lambda} \zeta}\right)^{2}\right]\right)$

$\beta=\pi(2 \zeta-1)$

with $\alpha$ the angle between the $z$-axis and the new direction $\left(z_{\text {new }}\right)$ and $\beta$ the rotational angle in the $x-y$ plane (Fig. A.1), with $\zeta$ a random number between 0 and 1 . These angles are transformed back to the rest frame $(X, Y, Z)$. After the new direction is calculated the next step is determined using Eqs. (A.3) and (A.4). The photon continues its path until it reaches the outer boundary of the computational grid or has a negligible intensity defined by the user.

\section{A.2. Computation of the mean intensity}

The mean intensity is determined in each cell through summation of all photons passing through the grid cell. As each photon represents an amount of energy which diminishes as it traverses a (dense) medium, the energy added to the cell is the mean energy per second of the photon along the path $\Delta s$ traveling with the velocity of light. The mean energy density $u_{\lambda}(i R, i z)$ of the radiation field is then calculated by dividing the total summed energy by the volume $V$ of the gridcell $i R, i z$.

$u_{\lambda}(i R, i z)=\frac{1}{V(i R, i z)} \sum I_{i} \frac{\Delta s}{c} \frac{\left(1-\mathrm{e}^{-\Delta \tau}\right)}{\Delta \tau}$.

The mean intensity $J_{\lambda}(i R, i z)$ in the grid cell is directly related to the mean energy density $u_{\lambda}(i R, i z)$ according to

$J_{\lambda}(i R, i z)=\frac{c}{4 \pi} u_{\lambda}(i R, i z)$. 
The mean intensity is the only output needed in this application, so the above description of scattering is sufficient. If the code is to be applied for both intensity and polarization, the four Stokes parameters have to be calculated (e.g., Whitney 1991). This is in principle readily implemented in the code, but has not been done here.

\section{A.3. $\mathrm{H}_{2}$ and $\mathrm{CO}$ self-shielding}

Photo dissociation of $\mathrm{H}_{2}$ proceeds through absorptions in the Lyman and Werner bands in the 912-1100 ̊ region. About $10-15 \%$ of all excitations lead to the dissociation of $\mathrm{H}_{2}$. Since this process proceeds through line excitation the absorption lines become optically thick once the $\mathrm{H}_{2}$ column in each level reaches $\sim 10^{14} \mathrm{~cm}^{-2}$. As this is easily reached for the objects of interest in this paper, self-shielding of $\mathrm{H}_{2}$ has to be taken into account. Only a limited amount of continuum radiation can be absorbed by $\mathrm{H}_{2}$, leaving a large flux available for other species to be dissociated. The equation used to calculate the shielding due to $\mathrm{H}_{2}$ is taken from Draine \& Bertoldi (1996), their Eq. (37) is shown here as (A.13). The equation describes the self-shielding of the $\mathrm{H}_{2}$ molecule through the Lyman and Werner series:

$f_{\mathrm{s}}\left(\mathrm{H}_{2}\right)=\frac{0.965}{(1-x / \mathrm{b} 5)^{2}}+\frac{0.035}{\sqrt{1-x}} \times \mathrm{e}^{-8.5 \times 10^{-4} \sqrt{1+x}}$

with $x=\frac{N\left(\mathrm{H}_{2}\right)\left[\mathrm{cm}^{-2}\right]}{5 \times 10^{14}}$ and $b_{5}=\frac{b\left[\mathrm{~cm} \mathrm{~s}^{-1}\right]}{1 \times 10^{5}}$ and $f_{s}$ the shielding of the $\mathrm{UV}$ in the $\mathrm{H}_{2}$ lines. Since $\mathrm{H}_{2}$ dissociates through lines, only a fraction of the continuum in between $912-1110 \AA$ is absorbed.

This equation is valid for each photon package $j$ traveling through a gridcell since the formulae is defined as the drop in intensity of the radiation field due to the column of $\mathrm{H}_{2}$. First the mean value of the function $f_{\mathrm{s}}$, for each package during its step is calculated, where the mean is taken over the column density $N$ of the step:

$\bar{F}(j, i R, i z)=\frac{\int_{N 1}^{N 2} f_{\mathrm{s}}(j, i R, i z) \mathrm{d} N}{\int_{N 1}^{N 2} \mathrm{~d} N}$.

This then leads to a mean value for the grid cell through:

$F_{\text {cell }}(i R, i z)=\frac{\sum_{j=0}^{n p(i R, i z)} \bar{F}(j, i R, i z) \Delta s(j, i R, i z)}{\sum_{j=0}^{n p(i R, i z)} \Delta s(j, i R, i z)}$,

with $n p(i R, i z)$ the number of photon steps taken in the grid cell defined by $i R$ and $i z$ and $\Delta s$ the length of the step of the photon. The value of $F_{\text {cell }}$ is then tabulated for each cell and applied to the intensities between 912-1100 A, which suffered dust extinction only.

Among the other molecules, only $\mathrm{CO}$ is affected significantly by self-shielding. In the other cases the dust extinction combined with $\mathrm{H}_{2}$ extinction, if the dissociation is at similar wavelengths (e.g., $\mathrm{CN}$ photodissociation or $\mathrm{C}$ photoionization), is sufficient to describe the rates of ionization and dissociation. $\mathrm{CO}$ shielding is treated in a similar way as $\mathrm{H}_{2}$ with the difference that instead of using an analytical solution, the values are taken from a table by Lee et al. (1996), in which shielding factor is given as a function of $\mathrm{H}_{2}$ column density, $\mathrm{CO}$ column density and $A_{\mathrm{v}}$. Calculation of the shielding factor thus require the $\mathrm{CO}$ abundance in each grid cell. In principle, iteration on the chemistry and radiative transfer is needed to calculate the precise $\mathrm{CO}$ dissociation rates. In the case of circumstellar disks, a large column is reached for each single cell due to the extremely high total columns, especially in the inner parts of disks. This causes the stellar dissociation rates to depend only on the mean value of the $\mathrm{CO}$ abundance in each cell. A constant $\mathrm{CO}$ abundance of $\mathrm{CO} / \mathrm{H}_{2}=7 \times 10^{-5}$ is assumed when calculating the $\mathrm{CO}$ self-shielding. If one is interested in a medium for which the precise positional change in the $\mathrm{CO}$ shielding is important, the spatial grid near the boundary at which the flux enters has to be increased and the chemistry and radiative transfer have to be iterated. Only a small chemical network is needed for this purpose, since the $\mathrm{CO}$ abundance depends mostly on a few simple molecules and atoms.

\section{A.4. Sources of radiation}

Radiation can come from several sources. Discussed here are two sources of radiation: interstellar radiation and stellar radiation. Since all boundary conditions consist of a surface through which flux passes, there is a general description for the angle distribution of the photon packages. These angles,

$\Theta=\cos ^{-1} \sqrt{1-\zeta}$

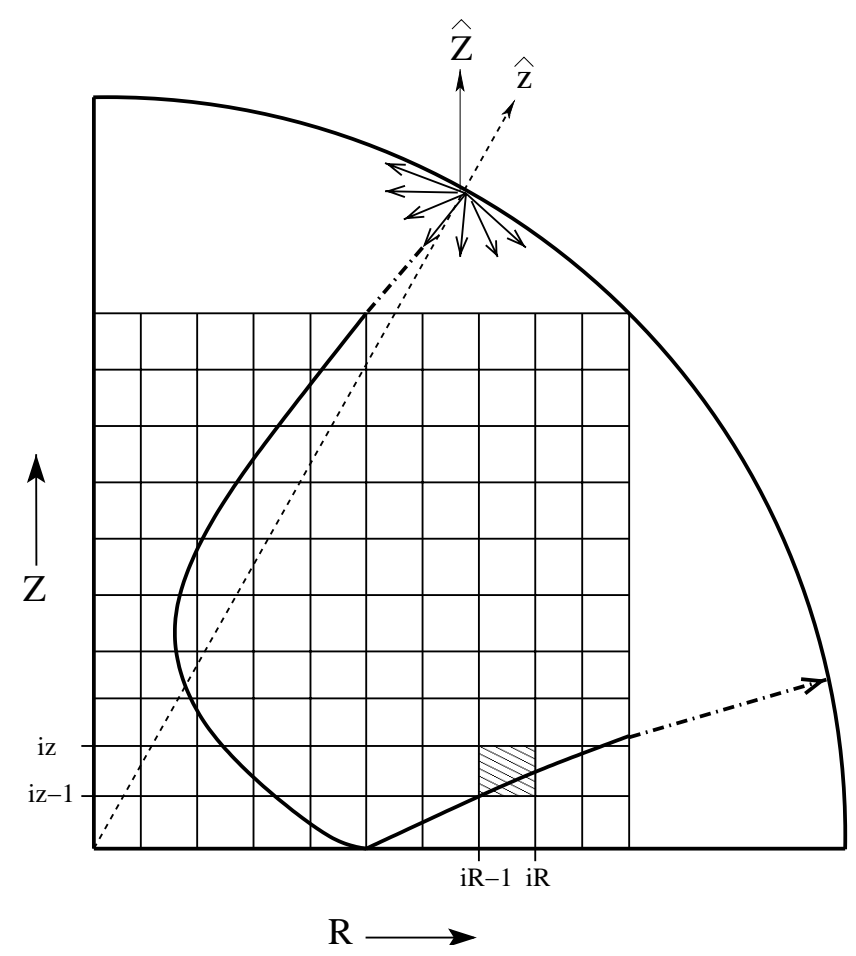

Fig. A.2. The grid represents the computational domain with a virtual shell around it. The dashed line denotes the the frame to describe the angle distribution of incoming photons from the boundary. The solid curved line is the path of the photon-package, the dot-dashed line is its virtual path. 
$\Phi=2 \pi \zeta$

are given in the frame in which the $z$-axis lies perpendicular to the tangent plane of the source surface. Equations (A.16) and (A.17) need to be transformed to calculate the angles in the plane where its $z$-axis is parallel to the $Z$-axis of the problem (Fig. A.2). The equations have been taken from Yang et al. (1995) and describe the isotropic emission from a surface.

- Interstellar field: the interstellar field is assumed to be isotropic over $4 \pi$ steradian while the computational domain is a rectangle in $R-z$ space (Fig. A.2). To calculate the mean intensity, a virtual sphere with a radius equal to that of the upper right corner is taken in order to represent the angle distribution of the incoming field. The starting positions of the photons are randomly taken at the surface of the sphere with a direction given by Eqs. (A.16) and (A.17). These directions are in the frame denoted by $\hat{z}$. For the calculation, the directions are transformed to the computational frame denoted by $\hat{Z}$. The photon packages are placed at the edge of the computational rectangle after which the photons follow the routine described in Sect. A.1. At each grid cell $(i R, i z)$ through which the photon passes, the mean intensity is updated. In general the Draine interstellar radiation field with the van Dishoeck \& Black (1982) extension for $\lambda>2000 \AA$ is used which can be scaled by a factor if required.

- Stellar light: since the medium in our problems is generally much larger than the stellar radius, the star can safely be assumed to be a point source. However, the total energy emitted does depend on the radius of the star. In this paper we assumed $R_{\star}=2 R_{\odot}$ The far-field expression for the mean intensity in a medium with negligible absorption is,

$$
J_{\lambda}(r)=\frac{I_{\lambda}}{4}\left(\frac{R_{*}}{r}\right)^{2},
$$

where $I_{\lambda}$ is the intensity at the surface of the star. It is a good approximation in the region of $R \gtrsim 5 R_{*}$, which we are concerned with. For problems where the mean intensities close to the star are important, emission from the real stellar surface should be taken into account.

\section{References}

Aikawa, Y., Miyama, S. M., Nakano, T., \& Umebayashi, T. 1996, ApJ, 467, 684

Aikawa, Y., Umebayashi, T., Nakano, T., \& Miyama, S. M. 1997, ApJ, 486, L51

Aikawa, Y., \& Herbst, E. 1999, A\&A, 351, 233

Aikawa, Y., van Zadelhoff, G. J., van Dishoeck, E. F., \& Herbst, E. 2002, A\&A, 386, 622

Beckwith, S. V. W., \& Sargent, A. I. 1996, Nature, 383, 139

Bell, K. R., Cassen, P. M., Klahr, H. H., \& Henning, T. 1997, ApJ, 486,372

Boissé, P. 1990, A\&A, 228, 483

Chiang, E. I., \& Goldreich, P. 1997, ApJ, 490, 368

Chiang, E. I., \& Goldreich, P. 1999, ApJ, 519, 279

Costa, V. M., Lago, M. T. V. T., Norci, L., \& Meurs, E. J. A. 2000, A\&A, 354, 621

D’Alessio, P., Cantó, J., Calvet, N., \& Lizano, S. 1998, ApJ, 500, 411
D’Alessio, P., Calvet, N., Hartmann, L., Lizano, S., \& Cantó, J. 1999, ApJ, 527, 893

Draine, B. T. 1978, ApJS, 36, 595

Draine, B. T., \& Bertoldi, F. 1996, ApJ, 468, 269

Dullemond, C. P., van Zadelhoff, G. J., \& Natta, A. 2002, A\&A, 389, 464

Dutrey, A., Guilloteau, S., Duvert, G., et al. 1996, A\&A, 309, 493

Dutrey, A., Guilloteau, S., \& Guélin, M. 2000, in Astrochemistry: From Molecular Clouds to Planetary Systems, ed. Y. C. Minh, \& E. F. van Dishoeck, IAU 197, 415

Gordon, K. D., Misselt, K. A., Witt, A. N., \& Clayton, G. C. 2001, ApJ, 551, 269

Hayashi, C. 1981, Prog. Theor. Phys. Suppl. 70, 35

Henyey, L. G., \& Greenstein, J. L. 1941, ApJ, 93, 70

Herbig, G. H., \& Goodrich, R. W. 1986, ApJ, 309, 294

Hogerheijde, M. R., \& van der Tak, F. F. S. 2000, A\&A, 362, 697

Jansen, D. J., Spaans, M., Hogerheijde, M. R., \& van Dishoeck, E. F. 1995a, A\&A, 303, 541

Jansen, D. J., van Dishoeck, E. F., Black, J. H., Spaans, M., \& Sosin, C. 1995b, A\&A, 302, 223

Kamp, I., \& van Zadelhoff, G. J. 2001, A\&A, 373, 641

Kenyon, S. J., \& Hartmann, L. 1987, ApJ, 323, 714

Lee, H.-H., Herbst, E., Pineau des Forêts, G., Roueff, E., \& Le Bourlot, J. 1996, A\&A, 311, 690

Markwick, A. J., Ilgner, M., Millar, T. J., \& Henning, T. 2002, A\&A, 385,632

Men'shchikov, A. B., \& Henning, T. 1997, A\&A, 318, 879

Nakagawa, Y., Nakazawa, K., \& Hayashi, C. 1981, Icarus, 45, 517

Nomura, H. 2002, ApJ, 567, 587

Osamura, Y., Fukuzawa, K., Terzieva, R., \& Herbst, E. 1999, ApJ, 519,697

Qi, C. 2001, Ph.D. Thesis, California Institute of Technology, Pasadena, California

Roberge, W. G., Jones, D., Lepp, S., \& Dalgarno, A. 1991, ApJS, 77, 287

Savage, B. D., Drake, J. F., Budich, W., \& Bohlin, R. C. 1977, ApJ, 216, 291

Simon, M., Dutrey, A., \& Guilloteau, S. 2000, ApJ, 545, 1034

Spaans, M. 1996, A\&A, 307, 271

Sternberg, A., \& Dalgarno, A. 1995, ApJS, 99, 565

Terzieva, R., \& Herbst, E. 1998, ApJ, 501, 207

Thi, W. F., van Zadelhoff, G. J., van Dishoeck, E. F., et al. 2002, A\&A, in preparation

van Dishoeck, E. F., \& Black, J. H. 1982, ApJ, 258, 533

van Dishoeck, E. F. 1988, in Rate Coefficients in Astrochemistry, ed. T. J. Millar, \& D. A. Williams (Kluwer, Astrophys. Space Science Library), 146, 49

van Dishoeck, E. F., \& Black, J. H. 1988, ApJ, 334, 771

van Zadelhoff, G.-J., van Dishoeck, E. F., Thi, W. F., \& Blake, G. A. 2001, A\&A, 377, 566

van Zadelhoff, G. J., Dullemond, C. P., van der Tak, F. F. S., et al. 2002, A\&A, submitted

Weingartner, J. C., \& Draine, B. T. 2001, ApJ, 548, 296

Weidenschilling, S. J. 1997, Icarus, 127, 290

Whitney, B. A., \& Hartmann, L. 1992, ApJ, 395, 529

Whitney, B. A. 1991, ApJS, 75, 1293

Witt, A. N. 1977, ApJS, 35, 1

Willacy, K., Klahr, H. H., Millar, T. J., \& Henning, Th. 1998, A\&A, 338,995

Willacy, K., \& Langer, W. D. 2000, ApJ, 544, 903

Yang, W. J., Taniguchi, H., \& Kudo, K. 1995, Radiative Heat Transfer by the Monte Carlo Method (San Diego: Academic Press) 